\title{
Error estimates of a linear decoupled Euler-FEM scheme for a mass diffusion model
}

\author{
Francisco Guillén-González • \\ Juan Vicente Gutiérrez-Santacreu
}

\begin{abstract}
We present error estimates of a linear fully discrete scheme for a threedimensional mass diffusion model for incompressible fluids (also called KazhikhovSmagulov model). All unknowns of the model (velocity, pressure and density) are approximated in space by $C^{0}$-finite elements and in time an Euler type scheme is used decoupling the density from the velocity-pressure pair. If we assume that the velocity and pressure finite-element spaces satisfy the inf-sup condition and the density finite-element space contains the products of any two discrete veloci-ties, we first obtain point-wise stability estimates for the density, under the constraint $\lim _{(h, k) \rightarrow 0} h / k=0$ ( $h$ and $k$ being the space and time discrete parameters, respectively), and error estimates for the velocity and density in energy type norms, at the same time. Afterwards, error estimates for the density in stronger norms are deduced. All these error estimates will be optimal (of order $\mathcal{O}(h+k)$ ) for regular enough solu-tions without imposing nonlocal compatibility conditions at the initial time. Finally, we also study two convergent iterative methods for the two problems to solve at each time step, which hold constant matrices (independent of iterations).
\end{abstract}

Mathematics Subject Classification (2000) 35Q35 $\cdot 65 \mathrm{M} 12 \cdot 65 \mathrm{M} 60$

This work has been partially supported by DGI-MEC (Spain), Grant MTM2006-07932 and P06-FQM-02373 (Junta de Andalucía).

F. Guillén-González (凶)

Dpto. E.D.A.N., University of Sevilla, Aptdo. 1160, 41080 Seville, Spain e-mail: guillen@us.es

J. V. Gutiérrez-Santacreu

Dpto. Matemática Aplicada I, Universidad de Sevilla, E.T.S.I. Informática,

Avda. Reina Mercedes, s/n., 41012 Seville, Spain

e-mail: juanvi@us.es 


\section{Introduction}

\subsection{Model}

Let $\Omega \subseteq \mathbb{R}^{d}$ ( $d=2$ or 3 ) be an open, bounded set with regular enough boundary $\Gamma$. Let $T>0$ and let $[0, T]$ be the time interval. We denote $Q=\Omega \times(0, T), \Sigma=\Gamma \times(0, T)$ and $\boldsymbol{n}$ the outwards unit normal vector to $\Gamma$.

We consider the system of equations governing the mixture of two miscible fluids with mass diffusion effect, the so-called mass diffusion model or KazhikhovSmagulov model [1,13]. The unknowns for this model are: $\boldsymbol{u}: Q \rightarrow \mathbb{R}^{d}$ the incompressible velocity field, $q: Q \rightarrow \mathbb{R}$ a potential function (related to the pressure) and $\rho: Q \rightarrow \mathbb{R}$ the fluid density, verifying the following partial differential equations:

$$
\begin{gathered}
\rho \boldsymbol{u}_{t}+((\rho \boldsymbol{u}-\lambda \nabla \rho) \cdot \nabla) \boldsymbol{u}-\mu \Delta \boldsymbol{u}-\lambda(\boldsymbol{u} \cdot \nabla) \nabla \rho+\nabla q=\rho \boldsymbol{f} \quad \text { in } Q, \\
\nabla \cdot \boldsymbol{u}=0 \quad \text { in } Q, \quad \rho_{t}+\boldsymbol{u} \cdot \nabla \rho-\lambda \Delta \rho=0 \quad \text { in } Q,
\end{gathered}
$$

where $f: Q \rightarrow \mathbb{R}^{d}$ are the external forces, and $\mu>0, \lambda>0$ are the viscosity and mass diffusion coefficients, respectively.

System (1)-(2) can be derived by assuming that the velocity $\boldsymbol{v}$ of a compressible Navier-Stokes system can be decomposed into $\boldsymbol{v}=\boldsymbol{u}-\lambda \nabla \log \rho$ with $\nabla \cdot \boldsymbol{u}=0$ (i.e. it is the sum of an incompressible part $\boldsymbol{u}$ and a potential part $-\lambda \nabla \log \rho$ ) and eliminating the $\lambda^{2}$-terms (see [1,9]), which is justified because of $\lambda$ is small in practical situations.

By decomposing the term involving second-order derivatives for the density as

$$
-\lambda(\boldsymbol{u} \cdot \nabla) \nabla \rho=-\lambda \nabla(\boldsymbol{u} \cdot \nabla \rho)+\lambda \nabla \cdot\left(\rho(\nabla \boldsymbol{u})^{t}\right)
$$

and defining $p=q-\lambda \boldsymbol{u} \cdot \nabla \rho$ (a modified potential function), the momentum system (1) reads

$$
\rho \boldsymbol{u}_{t}+((\rho \boldsymbol{u}-\lambda \nabla \rho) \cdot \nabla) \boldsymbol{u}-\mu \Delta \boldsymbol{u}+\lambda \nabla \cdot\left(\rho(\nabla \boldsymbol{u})^{t}\right)+\nabla p=\rho \boldsymbol{f} \quad \text { in } Q .
$$

System (2)-(3) is completed with the boundary conditions

$$
\boldsymbol{u}_{\left.\right|_{\Sigma}}=0,\left.\quad \frac{\partial \rho}{\partial \boldsymbol{n}}\right|_{\Sigma}=0
$$

and the initial conditions

$$
\rho(\boldsymbol{x}, 0)=\rho_{0}(\boldsymbol{x}), \quad \boldsymbol{u}(\boldsymbol{x}, 0)=\boldsymbol{u}_{0}(\boldsymbol{x}), \quad \boldsymbol{x} \in \Omega,
$$

where $\rho_{0}: \Omega \rightarrow \mathbb{R}^{+}$and $\boldsymbol{u}_{0}: \Omega \rightarrow \mathbb{R}^{d}$ are given functions.

Throughout this work, we always assume the following hypothesis on the initial density:

$$
0<m \leq \rho_{0}(\boldsymbol{x}) \leq M \quad \text { in } \Omega
$$




\subsection{Notation and functional spaces}

As usual $L^{p}(\Omega)$ denotes the space of p-summable functions in $\Omega$, and $\|\cdot\|_{L^{p}(\Omega)}$ its norm. We denote the inner-product in $L^{2}$ by $(\cdot, \cdot)$ and by $\|\cdot\|_{L^{2}(\Omega)}=|\cdot|$ its norm. We denote the classic Sobolev spaces $W^{k, p}(\Omega)$ and $W_{0}^{k, p}(\Omega)\left(\right.$ or $H^{k}(\Omega)$ and $H_{0}^{k}(\Omega)$, respectively, for $p=2)$, with $p \geq 1$ and $k \in \mathbb{N}$, and $\|\cdot\|_{W^{k, p}(\Omega)}$ its norm $\left(\|\cdot\|_{H^{k}(\Omega)}\right.$ for $p=2$ ). We will use frequently the semi-norm of the gradient $|\nabla u|$ as norm for $u \in H_{0}^{1}(\Omega)$. We will use bold-face letter for vectorial spaces and their elements.

Next, we will describe briefly the usual functional spaces in the framework of fluid mechanics:

$$
\begin{aligned}
\boldsymbol{H} & =\left\{\boldsymbol{u}: \boldsymbol{u} \in \boldsymbol{L}^{2}(\Omega), \nabla \cdot \boldsymbol{u}=0 \text { in } \Omega, \boldsymbol{u} \cdot \boldsymbol{n}=0 \text { on } \Gamma\right\}, \\
\boldsymbol{V} & =\left\{\boldsymbol{u}: \boldsymbol{u} \in \boldsymbol{H}_{0}^{1}(\Omega), \nabla \cdot \boldsymbol{u}=0 \text { in } \Omega\right\}, \\
L_{0}^{2}(\Omega) & =\left\{p: p \in L^{2}(\Omega), \int_{\Omega} p(\boldsymbol{x}) d \boldsymbol{x}=0\right\} .
\end{aligned}
$$

On the other hand, for the density we will consider the affine space

$$
H_{N}^{2}(\Omega)=\left\{\rho \in H^{2}(\Omega): \frac{\partial \rho}{\partial \boldsymbol{n}}=0 \text { on } \partial \Omega, \int_{\Omega} \rho(\boldsymbol{x})=\int_{\Omega} \rho_{0}(\boldsymbol{x})\right\} .
$$

Obviously, $H_{N}^{2}(\Omega)=\overline{\rho_{0}}+H_{N, 0}^{2}(\Omega)$, where $\overline{\rho_{0}}=\frac{1}{|\Omega|} \int_{\Omega} \rho_{0}(\boldsymbol{x}) d \boldsymbol{x}$ and

$$
H_{N, 0}^{2}(\Omega)=\left\{\rho \in H^{2}(\Omega): \frac{\partial \rho}{\partial \boldsymbol{n}}=0 \text { on } \partial \Omega, \int_{\Omega} \rho(\boldsymbol{x})=0\right\} .
$$

Therefore, $H_{N}^{2}(\Omega)$ is a affine space associated to $H_{N, 0}^{2}(\Omega)$. Accordingly to the $H^{2}$-regularity of the Poisson-Neumann problem, the norm $\|\rho\|_{H^{2}(\Omega)}$ is equivalent to the seminorm $\|\Delta \rho\|_{L^{2}(\Omega)}$ in $H_{N, 0}^{2}(\Omega)$.

\subsection{Known results}

Concerning model (2)-(5), Kazhikhov and Smagulov [1,13] proved, via a semiGalerkin method, the existence of global weak solutions under the following hypothesis on the viscosity and diffusion coefficients

$$
\lambda<2 \mu /(M-m)
$$

and the existence and uniqueness of local strong solutions (which is global in time in $2 D$ domains). On the other hand, Salvi [14] proved the existence of weak solutions in 
non-cylindrical domains. Secchi [17] studied the case $\Omega=\mathbb{R}^{3}$, proving the existence and uniqueness of strong solutions, by using a fixed-point argument.

With respect to a more complete model than (2)-(5) (adding to (3) $\lambda^{2}$-terms), Beirão da Veiga [2] and Secchi [16] established the local existence of strong solutions by means of a linearization and fixed point argument. In [16], Secchi proved the existence and uniqueness of global weak solutions in $2 D$ domains imposing $\lambda / \mu$ small enough and the asymptotic behavior, as $\lambda \rightarrow 0$, towards a weak solution of the density-dependent Navier-Stokes problem. Recently, in [8], by means of an iterative method, existence of regular solutions (and some error estimates) has been proved. Finally, see [15] for a recent exposition of theoretical results of this model, including the problem of the $L^{q}$-maximal regularity.

There are not many results concerning the numerical analysis of (2)-(5). Using a finite element method, two numerical schemes have been recently developed in [9] and [10] for (2)-(5) in the two- and three-dimensional case, respectively. For the twodimensional case, a numerical scheme is constructed being unconditionally stable and convergent towards the (unique) solution of the continuous problem. This scheme is obtained by applying a truncating operator in the terms depending on the density which require positiveness and pointwise bounds. In the three-dimensional case, a conditionally stable and convergent scheme is designed for which an approximate maximum principle is shown, bounding by excess and defect the approximate density with respect to the upper and lower bound of the initial density. For this, the convective velocity of the discrete density equation is projected onto a discrete free-divergence space related to the density space. An extension of the results in [10] for the complete model with $\lambda^{2}$-terms has been recently obtained in [11].

Both schemes of [9] and [10] are based on the backward Euler method in time, where the computation of the density and the velocity-pressure pair is decoupled at each time step, by means of two linear problems.

In [5], a numerical algorithm is developed by using a characteristic method in time and finite elements in space. The authors give optimal error order under certain restrictions on the discrete parameters and assuming regularity hypotheses on the exact solution, as for instance $\boldsymbol{u} \in L^{\infty}\left(0, T ; \boldsymbol{H}^{3}(\Omega)\right)$, which turn out to be more demanding than we will impose in this work. In particular, such a regularity requires a nonlocal compatibility condition for the data at $t=0$.

\subsection{Description of the scheme}

The scheme that we study in this work is based on the following weak mixed formulation of problem (2)-(5):

$$
\left\{\begin{array}{l}
\left(\rho \boldsymbol{u}_{t}, \overline{\boldsymbol{u}}\right)+((\rho \boldsymbol{u}-\lambda \nabla \rho) \cdot \nabla \boldsymbol{u}, \overline{\boldsymbol{u}})+\left(\mu \nabla \boldsymbol{u}-\lambda \rho(\nabla \boldsymbol{u})^{t}, \nabla \overline{\boldsymbol{u}}\right) \\
-(p, \nabla \cdot \overline{\boldsymbol{u}})=(\rho \boldsymbol{f}, \overline{\boldsymbol{u}}), \quad(\nabla \cdot \boldsymbol{u}, \bar{p})=0 \\
\left(\rho_{t}, \bar{\rho}\right)+(\boldsymbol{u} \cdot \nabla \rho, \bar{\rho})+\lambda(\nabla \rho, \nabla \bar{\rho})=0
\end{array}\right.
$$

for all $(\overline{\boldsymbol{u}}, \bar{p}, \bar{\rho}) \in \boldsymbol{H}_{0}^{1}(\Omega) \times L_{0}^{2}(\Omega) \times H^{1}(\Omega)$. We consider a backward first-order finite difference for the time derivative on a partition, which, for simplicity, we 
suppose uniform on $[0, T]$ with time step $k=T / N:\left(t_{n}=n k\right)_{n=0}^{n=N}$. To approximate the unknowns density, velocity and pressure, we will use finite element spaces denoted by $\left(W_{h}, \boldsymbol{V}_{h}, M_{h}\right)$, being conforming approximations of $\left(H^{1}, \boldsymbol{H}_{0}^{1}, L_{0}^{2}\right)$ and verifying hypotheses $(H 2)-(H 4)$ described in Sect. 2.1 below.

Under the foregoing statement, we propose the following numerical scheme.

Initialization: Let $\left(\boldsymbol{u}_{h}^{0}, \rho_{h}^{0}\right) \in \boldsymbol{V}_{h} \times W_{h}$ be approximations of $\left(\boldsymbol{u}_{0}, \rho_{0}\right)$, as $h \rightarrow 0$.

Time step $n+1$ : Given $\rho_{h}^{n} \in W_{h}$ and $\boldsymbol{u}_{h}^{n} \in \boldsymbol{V}_{h}$, find $\rho_{h}^{n+1} \in W_{h}$ such that for each $\bar{\rho}_{h} \in W_{h}:$

$$
\left(\frac{\rho_{h}^{n+1}-\rho_{h}^{n}}{k}, \bar{\rho}_{h}\right)+\left(\boldsymbol{u}_{h}^{n} \cdot \nabla \rho_{h}^{n+1}, \bar{\rho}_{h}\right)+\lambda\left(\nabla \rho_{h}^{n+1}, \nabla \bar{\rho}_{h}\right)=0 .
$$

Given $\rho_{h}^{n}, \rho_{h}^{n+1} \in W_{h}$ and $\boldsymbol{u}_{h}^{n} \in \boldsymbol{V}_{h}$, find $\left(\boldsymbol{u}_{h}^{n+1}, p_{h}^{n+1}\right) \in \boldsymbol{V}_{h} \times M_{h}$ such that for each $\left(\overline{\boldsymbol{u}}_{h}, \bar{p}_{h}\right) \in \boldsymbol{V}_{h} \times M_{h}$ :

$$
\left\{\begin{array}{c}
\left(\rho_{h}^{n} \frac{\boldsymbol{u}_{h}^{n+1}-\boldsymbol{u}_{h}^{n}}{k}, \overline{\boldsymbol{u}}_{h}\right)+\left(\left(\left(\rho_{h}^{n+1} \boldsymbol{u}_{h}^{n}-\lambda \nabla \rho_{h}^{n+1}\right) \cdot \nabla\right) \boldsymbol{u}_{h}^{n+1}, \overline{\boldsymbol{u}}_{h}\right)+a\left(\rho_{h}^{n+1}, \boldsymbol{u}_{h}^{n+1}, \overline{\boldsymbol{u}}_{h}\right) \\
+\frac{1}{2}\left(\left(\nabla \cdot \boldsymbol{u}_{h}^{n}\right) \rho_{h}^{n+1} \boldsymbol{u}_{h}^{n+1}, \overline{\boldsymbol{u}}_{h}\right)=\left(\rho_{h}^{n+1} \boldsymbol{f}\left(t_{n+1}\right), \overline{\boldsymbol{u}}_{h}\right)+\left(p_{h}^{n+1}, \nabla \cdot \overline{\boldsymbol{u}}_{h}\right), \\
\left(\nabla \cdot \boldsymbol{u}_{h}^{n+1}, \bar{p}_{h}\right)=0,
\end{array}\right.
$$

where

$$
a(\rho, \boldsymbol{u}, \boldsymbol{v})=\mu(\nabla \boldsymbol{u}, \nabla \boldsymbol{v})-\lambda \int_{\Omega}\left(\rho-\frac{\widetilde{M}+\widetilde{m}}{2}\right)(\nabla \boldsymbol{u})^{t}: \nabla \boldsymbol{v} d \boldsymbol{x}
$$

with

$$
\widetilde{M}>M, \quad 0<\widetilde{m}<m \quad \text { such that } \quad \lambda \frac{\widetilde{M}-\widetilde{m}}{2}<\mu .
$$

Note that this choice of $\widetilde{M}$ and $\widetilde{m}$ is possible owing to hypothesis (7). The trilinear form $a(\cdot, \cdot, \cdot)$ satisfies the following properties: if $0<\widetilde{m} \leq \rho \leq \widetilde{M}$, then

$$
\begin{array}{ll}
a(\rho, \boldsymbol{u}, \boldsymbol{u}) \geq \frac{\mu_{1}}{2}|\nabla \boldsymbol{u}|^{2} \text { where } \frac{\mu_{1}}{2}=\mu-\lambda \frac{\widetilde{M}-\widetilde{m}}{2}(>0), & \text { (coercivity) } \\
a(\rho, \boldsymbol{u}, \boldsymbol{v}) \leq C|\nabla \boldsymbol{u}||\nabla \boldsymbol{v}| & \quad \text { (continuity). }
\end{array}
$$

Note that the numerical scheme presented here is implicit with respect to the linear terms and semi-implicit with respect to the nonlinear terms so that it has allowed us to design a linear scheme which decouples the computation of $\rho_{h}^{n+1}$ and $\left(\boldsymbol{u}_{h}^{n+1}, p_{h}^{n+1}\right)$.

The well-posedness of the linear convective-diffusion problem (9) is standard. However, some care is required to assure that the linear mixed problem (10)-(11) is well-posed. Besides the well-known Brezzi-Babuska or Inf-Sup stability condition 
(see $(H 3)$ below), we must assure $\rho_{h}^{n}>0$, which we are going to get by induction later on (see Corollary 7). In fact, the existence (and uniqueness) of (10)-(11) will be also proved by induction at the same time that weak error estimates (see Theorem 9).

Remark 1 The choice of approximating $\left(\rho \boldsymbol{u}_{t}\right)\left(t_{n+1}\right)$ as $\rho_{h}^{n} \frac{\boldsymbol{u}_{h}^{n+1}-\boldsymbol{u}_{h}^{n}}{k}$ is justified by the following equality $[9,10]$ :

$$
\begin{gathered}
\left(\rho_{h}^{n} \frac{\boldsymbol{u}_{h}^{n+1}-\boldsymbol{u}_{h}^{n}}{k}, \boldsymbol{u}_{h}^{n+1}\right)+\frac{1}{2}\left(\frac{\rho_{h}^{n+1}-\rho_{h}^{n}}{k}, \boldsymbol{u}_{h}^{n+1} \cdot \boldsymbol{u}_{h}^{n+1}\right) \\
=\frac{1}{2} \frac{\rho_{h}^{n+1}\left|\boldsymbol{u}_{h}^{n+1}\right|^{2}-\rho_{h}^{n}\left|\boldsymbol{u}_{h}^{n}\right|^{2}}{k}+\frac{1}{2} k \rho_{h}^{n}\left|\frac{\boldsymbol{u}_{h}^{n+1}-\boldsymbol{u}_{h}^{n}}{k}\right|^{2} .
\end{gathered}
$$

This is the discrete version of the following continuous equality on the time derivative of the kinetic energy:

$$
\left(\rho \frac{d}{d t} \boldsymbol{u}, \boldsymbol{u}\right)+\frac{1}{2}\left(\frac{d}{d t} \rho, \boldsymbol{u} \cdot \boldsymbol{u}\right)=\frac{1}{2} \int_{\Omega} \frac{d}{d t}\left(\rho|\boldsymbol{u}|^{2}\right) .
$$

Comparing the schemes developed in [9] and [10] with scheme (9)-(11), we can remark the following similarities and differences.

1. In [10] the velocity $\boldsymbol{u}_{h}^{n}$ of the convective term of (9) is replaced by the $H^{1}$-projected velocity $\boldsymbol{w}_{h}^{n}$ onto a discrete free-divergence space, namely $\boldsymbol{w}_{h}^{n} \in \widetilde{\boldsymbol{V}_{h}}$ (jointly with $q_{h}^{n} \in \widetilde{M}_{h}$ ) such that

$$
\left\{\begin{aligned}
\left(\nabla\left(\boldsymbol{w}_{h}^{n}-\boldsymbol{u}_{h}^{n}\right), \nabla \overline{\boldsymbol{w}}_{h}\right)-\left(q_{h}^{n}, \nabla \cdot \overline{\boldsymbol{w}}_{h}\right)=0 \quad \forall \overline{\boldsymbol{w}}_{h} \in \widetilde{\boldsymbol{V}}_{h}, \\
\left(\nabla \cdot \boldsymbol{w}_{h}^{n}, \bar{q}_{h}\right)=0 \quad \forall \bar{q}_{h} \in \widetilde{M}_{h},
\end{aligned}\right.
$$

where $\left(\widetilde{\boldsymbol{V}}_{h}, \widetilde{M}_{h}\right)$ satisfies the inf-sup condition, $\left(W_{h} \cdot W_{h}\right) \cap L_{0}^{2}(\Omega) \subset \widetilde{M}_{h}$ and $M_{h} \subset \widetilde{M}_{h}$. Obviously, such a projection can be avoided by selecting $\boldsymbol{V}_{h}=\widetilde{\boldsymbol{V}}_{h}$, and $M_{h}=\widetilde{M}_{h}$.

2. Also, in the discrete momentum system (10), the stabilization term $\frac{1}{2}\left(\left(\nabla \cdot \boldsymbol{u}_{h}^{n}\right) \rho_{h}^{n+1} \boldsymbol{u}_{h}^{n+1}, \overline{\boldsymbol{u}}_{h}\right)$ is replaced in [10] by

$$
\frac{1}{2}\left(\frac{\rho_{h}^{n+1}-\rho_{h}^{n}}{k}, \boldsymbol{u}_{h}^{n+1} \cdot \overline{\boldsymbol{u}}_{h}\right)-\frac{1}{2}\left(\rho_{h}^{n+1} \boldsymbol{u}_{h}^{n}-\lambda \nabla \rho_{h}^{n+1}, \nabla\left(\boldsymbol{u}_{h}^{n+1} \cdot \overline{\boldsymbol{u}}_{h}\right)\right) .
$$

It turns out easy to obverse that under the hypothesis $\boldsymbol{V}_{h} \cdot \boldsymbol{V}_{h} \subset W_{h}$ imposed in (H4) (see Sect. 2.1), the two above stabilization terms coincides. Indeed, take 
$\bar{\rho}_{h}=\frac{1}{2} \boldsymbol{u}_{h}^{n+1} \cdot \overline{\boldsymbol{u}}_{h}$ in (9) and integrate by parts in the convective term to find

$$
\begin{aligned}
& \frac{1}{2}\left(\frac{\rho_{h}^{n+1}-\rho_{h}^{n}}{k}, \boldsymbol{u}_{h}^{n+1} \cdot \overline{\boldsymbol{u}}_{h}\right)-\frac{1}{2}\left(\rho_{h}^{n+1} \boldsymbol{u}_{h}^{n}-\lambda \nabla \rho_{h}^{n+1}, \nabla\left(\boldsymbol{u}_{h}^{n+1} \cdot \overline{\boldsymbol{u}}_{h}\right)\right) \\
& \quad=\frac{1}{2}\left(\left(\nabla \cdot \boldsymbol{u}_{h}^{n}\right) \rho_{h}^{n+1} \boldsymbol{u}_{h}^{n+1}, \overline{\boldsymbol{u}}_{h}\right) .
\end{aligned}
$$

Therefore, (10) coincides with the discrete momentum system in [10].

3. But the hypothesis $\boldsymbol{V}_{h} \cdot \boldsymbol{V}_{h} \subset W_{h}$ imposed in $(H 4)$ and the hypothesis $\left(W_{h} \cdot W_{h}\right) \cap L_{0}^{2}(\Omega) \subset \widetilde{M}_{h}$ imposed in [10] are, in a certain sense, opposite. Indeed, in the case to avoid the projection step, we select $\boldsymbol{V}_{h}=\widetilde{\boldsymbol{V}}_{h}$ and $M_{h}=\widetilde{M}_{h}$. Then, one arrives at

$$
\left(\boldsymbol{V}_{h} \cdot \boldsymbol{V}_{h} \cdot \boldsymbol{V}_{h} \cdot \boldsymbol{V}_{h}\right) \subset\left(W_{h} \cdot W_{h}\right) \subset M_{h} \subset \boldsymbol{V}_{h}
$$

(the last inclusion is due to the inf-sup condition for $\left.\left(\boldsymbol{V}_{h}, M_{h}\right)\right)$ which are contradictory inclusions.

4. In the scheme given in [9] for $2 D$ domains, the semi-implicit convective term $\left(\boldsymbol{u}_{h}^{n} \cdot \nabla \rho_{h}^{n+1}, \bar{\rho}_{h}\right)$ in (9) is replaced by the fully explicit term $\left(\boldsymbol{u}_{h}^{n} \cdot \nabla \rho_{h}^{n}, \bar{\rho}_{h}\right)$, the following stabilization terms

$$
\frac{1}{2}\left(\frac{\left[\rho_{h}^{n+1}\right]_{T}-\left[\rho_{h}^{n}\right]_{T}}{k}, \boldsymbol{u}_{h}^{n+1} \cdot \overline{\boldsymbol{u}}_{h}\right)-\frac{1}{2}\left(\rho_{h}^{n+1} \boldsymbol{u}_{h}^{n}-\lambda \nabla \rho_{h}^{n+1}, \nabla\left(\boldsymbol{u}_{h}^{n+1} \cdot \overline{\boldsymbol{u}}_{h}\right)\right),
$$

are introduced instead of $\frac{1}{2}\left(\left(\nabla \cdot \boldsymbol{u}_{h}^{n}\right) \rho_{h}^{n+1} \boldsymbol{u}_{h}^{n+1}, \overline{\boldsymbol{u}}_{h}\right)$ in the momentum system (10), where $[\cdot]_{T}$ is a truncating operator by nodes between the upper and lower bounds of the initial density, and the same truncating operator is considered in the discrete momentum system in the terms depending on the discrete density which requires positiveness and pointwise bounds for density. Accordingly, (10) is different to the discrete momentum system developed in [9].

An important observation on scheme (9)-(11) is that it is not clear how to obtain a priori estimates without imposing regularity hypotheses on the exact solution, unlike the schemes developed in $[9,10]$, which are stables (and convergent towards weak solutions). On the contrary, to prove error estimates for the stable schemes studied in $[9,10]$ introduces important difficulties, with respect to the scheme (9)-(11) introduced here.

\subsection{Main results of this paper}

In this paper, we denote by $C$ a generic positive constant (which may vary in each bound) depending on the continuous solution, say $(\boldsymbol{u}, p, \rho)$, and the fixed parameters 
of the problem $(\lambda, \mu)$. When necessary, we use $C_{s}, G_{i}$ and $A$ to denote particular positive constants.

By denoting the errors between the continuous and discrete solution in time at $t=t_{n}$ as:

$$
e_{u}^{n}=\boldsymbol{u}_{h}^{n}-\boldsymbol{u}\left(t_{n}\right), \quad e_{p}^{n}=p_{h}^{n}-p\left(t_{n}\right), \quad e_{\rho}^{n}=\rho_{h}^{n}-\rho\left(t_{n}\right)
$$

we will prove the following results.

Theorem 2 Assume hypotheses (H0)-(H4) (see Sect. 2.1 below) and the constraint

$$
\lim _{(h, k) \rightarrow 0} \frac{h}{k}=0 .
$$

Then, there exists a unique solution $\left(\rho_{h}^{n}, \boldsymbol{u}_{h}^{n}, p_{h}^{n}\right)$ of scheme (9)-(11) and the following error estimates hold, for $h$ and $k$ small enough:

$$
\left\{\begin{array}{l}
\max _{0 \leq n \leq N-1}\left(\widetilde{m}\left|\boldsymbol{e}_{\boldsymbol{u}}^{n+1}\right|^{2}+A\left|e_{\rho}^{n+1}\right|^{2}\right)+\sum_{n=0}^{N-1}\left(\frac{\widetilde{m}}{2}\left|\boldsymbol{e}_{\boldsymbol{u}}^{n+1}-\boldsymbol{e}_{\boldsymbol{u}}^{n}\right|^{2}+\frac{A}{2}\left|e_{\rho}^{n+1}-e_{\rho}^{n}\right|^{2}\right) \\
+k \sum_{n=0}^{N-1}\left(\frac{\mu_{1}}{2}\left|\nabla \boldsymbol{e}_{\boldsymbol{u}}^{n+1}\right|^{2}+A \lambda\left|\nabla e_{\rho}^{n+1}\right|^{2}\right) \leq C\left(k^{2}+h^{2}\right),
\end{array}\right.
$$

where $A>0$ is a constant independent of $(h, k)$.

Theorem 3 Under conditions of Theorem 2 and $\rho_{t} \in L^{2}\left(0, T ; H^{2}(\Omega)\right)$, the following error estimates hold for $h$ and $k$ small enough:

$$
\begin{aligned}
& \max _{0 \leq n \leq N}\left|\nabla e_{\rho}^{n+1}\right|^{2}+\sum_{n=0}^{N-1}\left|\nabla\left(e_{\rho}^{n+1}-e_{\rho}^{n}\right)\right|^{2}+k \sum_{n=0}^{N-1}\left|P_{h}\left(\Delta \rho\left(t_{n+1}\right)\right)-\Delta_{h} \rho_{h}^{n+1}\right|^{2} \\
& \leq C\left(k^{2}+h^{2}\right)
\end{aligned}
$$

where $\Delta_{h} \rho_{h}^{n+1}$ is the discrete Laplacian of $\rho_{h}^{n+1}$ defined in (54) and $P_{h}$ is the $L^{2}$-orthogonal projection onto $W_{h}$.

Note that if $\rho \in L^{2}\left(0, T ; H^{3}(\Omega)\right)$ holds, one gets the following total error estimate for the density

$$
k \sum_{n=0}^{N-1}\left|\Delta \rho\left(t_{n+1}\right)-\Delta_{h} \rho_{h}^{n+1}\right|^{2} \leq C\left(k^{2}+h^{2}\right) .
$$

Finally, in this work, we analyze two iterative methods to approximate problems (9) and (11), with constant matrices by iteration. Theses scheme are described as follows:

Iterative method for problem (9). Given $\left(\rho_{h}^{n}, \boldsymbol{u}_{h}^{n}\right)$, the solution $\rho_{h}^{n+1}$ to (9) is approximated by the sequence $\left(\rho_{h}^{n+1, i}\right)_{i}$ defined as: 
Initialization: Let $\rho_{h}^{n+1,0}=\rho_{h}^{n}$.

Step $i+1$ : Given $\rho_{h}^{n+1, i}$, find $\rho_{h}^{n+1, i+1} \in W_{h}$ such that for each $\bar{\rho}_{h} \in W_{h}$ :

$$
\left(\frac{\rho_{h}^{n+1, i+1}-\rho_{h}^{n}}{k}, \bar{\rho}_{h}\right)+\lambda\left(\nabla \rho_{h}^{n+1, i+1}, \nabla \bar{\rho}_{h}\right)=-\left(\boldsymbol{u}_{h}^{n} \cdot \nabla \rho_{h}^{n+1, i}, \bar{\rho}_{h}\right) .
$$

Iterative method for problem (10)-(11). Given $\left(\rho_{h}^{n}, \rho_{h}^{n+1}, \boldsymbol{u}_{h}^{n}\right)$, the solution $\left(\boldsymbol{u}_{h}^{n+1}\right.$, $\left.p_{h}^{n+1}\right)$ to (10)-(11) is approximated by the sequence $\left(\boldsymbol{u}_{h}^{n+1, i}, p_{h}^{n+1, i}\right)_{i}$ defined as: Initialization: Let $\boldsymbol{u}_{h}^{n+1,0}=\boldsymbol{u}_{h}^{n}$.

Step $i+1$ : Given $\boldsymbol{u}_{h}^{n+1, i}$, find $\left(\boldsymbol{u}_{h}^{n+1, i+1}, p_{h}^{n+1, i+1}\right) \in \boldsymbol{V}_{h} \times M_{h}$ such that for each $\left(\overline{\boldsymbol{u}}_{h}, \bar{p}_{h}\right) \in \boldsymbol{V}_{h} \times M_{h}$ :

$$
\left\{\begin{array}{c}
\left(\rho_{\bar{m}}^{\widetilde{M}} \frac{\boldsymbol{u}_{h}^{n+1, i+1}-\boldsymbol{u}_{h}^{n}}{k}, \overline{\boldsymbol{u}}_{h}\right)+\mu\left(\nabla \boldsymbol{u}_{h}^{n+1, i+1}, \nabla \overline{\boldsymbol{u}}_{h}\right)-\left(p_{h}^{n+1, i+1}, \nabla \cdot \overline{\boldsymbol{u}}_{h}\right) \\
=-\left(\left(\left(\rho_{h}^{n+1} \boldsymbol{u}_{h}^{n}-\lambda \nabla \rho_{h}^{n+1}\right) \cdot \nabla\right) \boldsymbol{u}_{h}^{n+1, i}, \overline{\boldsymbol{u}}_{h}\right)-\lambda \int_{0}^{T}\left(\rho_{\bar{m}}^{\widetilde{M}}-\rho_{h}^{n+1}\right)\left(\nabla \boldsymbol{u}_{h}^{n+1, i}\right)^{t}: \nabla \overline{\boldsymbol{u}}_{h} \\
-\frac{1}{2}\left(\nabla \cdot \boldsymbol{u}_{h}^{n} \rho_{h}^{n+1} \boldsymbol{u}_{h}^{n+1, i}, \overline{\boldsymbol{u}}_{h}\right)+\left(\rho_{h}^{n+1} \boldsymbol{f}^{n+1}, \overline{\boldsymbol{u}}_{h}\right)+\left(\left(\rho_{\bar{m}}^{\widetilde{M}}-\rho_{h}^{n}\right) \frac{\boldsymbol{u}_{h}^{n+1, i}-\boldsymbol{u}_{h}^{n}}{k}, \overline{\boldsymbol{u}}_{h}\right) \\
\left(\nabla \cdot \boldsymbol{u}_{h}^{n+1, i}, \bar{p}_{h}\right)=0
\end{array}\right.
$$

where $\rho_{\widetilde{m}}^{\widetilde{M}}=\frac{\widetilde{M}+\widetilde{m}}{2}$. We will see the convergence of the approximations $\left(\boldsymbol{u}_{h}^{n+1, i}, p_{h}^{n+1, i}, \rho_{h}^{n+1, i}\right)$ towards $\left(\boldsymbol{u}_{h}^{n+1}, p_{h}^{n+1}, \rho_{h}^{n+1}\right)$ as $i \rightarrow \infty$, whenever $k$ is small enough.

The rest of the work is organized as follows. In Sect. 2 we describe the hypotheses about the domain, the data and the finite element approximation, and we define suitable interpolation operators. In Sect. 3 we prove, by an induction process at each time step, point-wise estimates for the discrete density which allow us to obtain in Sect. 4, the existence and uniqueness of a solution of scheme (9)-(11) and some convergence rates, firstly in energy norms for density and velocity (Theorem 2), and afterwards in a certain discrete stronger norm for the density (Theorem 3). Finally, in Sect. 5, we prove that the iterative methods (14) and (15)-(16) are well-posed and convergent.

\section{Preliminaries}

From now on, fix $\Omega$ an open, bounded set of $\mathbb{R}^{d}(d=2$ or 3 ) with Lipschitz-continuous polyhedral or polygonal boundary and let $\left\{\mathcal{T}_{h}\right\}_{h>0}$ be a family of triangulations such that $\bar{\Omega}=\bigcup_{K \in \mathcal{T}_{h}} K, h$ being the maximum diameter of elements of $\mathcal{T}_{h}$.

\subsection{Hypotheses}

(H0) Regularity for the data: Let $\boldsymbol{u}_{0} \in \boldsymbol{V} \cap\left(\boldsymbol{W}^{1,3}(\Omega) \cap \boldsymbol{L}^{\infty}(\Omega)\right), \rho_{0} \in H_{N}^{2}(\Omega)$ with $0<m \leq \rho_{0} \leq M$ in $\Omega$, and $\boldsymbol{f} \in L^{2}\left(0, T ; \boldsymbol{L}^{3}(\Omega)\right)$ with $\boldsymbol{f}_{t} \in L^{2}\left(0, T ; \boldsymbol{L}^{6 / 5}(\Omega)\right)$. 
Constraints on the parameters $(\lambda, \mu, m, M)$ : Assume that $\lambda \frac{M-m}{2}<\mu$ and let $\widetilde{m}, \widetilde{M}$ satisfying (12).

Regularity for the solution: Suppose that $(\rho, \boldsymbol{u}, p)$ is the unique solution to problem (2)-(5) in $(0, T)$ with the following regularity:

$$
\begin{aligned}
& \rho \in L^{\infty}\left(0, T ; W^{1,3}(\Omega) \cap L^{\infty}(\Omega)\right) \cap L^{2}\left(0, T ; H^{2}(\Omega)\right), \\
& \rho_{t} \in L^{2}\left(0, T, H^{1}(\Omega)\right), \\
& \boldsymbol{u} \in L^{\infty}\left(0, T ; \boldsymbol{W}^{1,3}(\Omega) \cap \boldsymbol{L}^{\infty}(\Omega)\right) \cap L^{2}\left(0, T ; \boldsymbol{H}^{2}(\Omega)\right), \\
& \boldsymbol{u}_{t} \in L^{\infty}\left(0, T, \boldsymbol{L}^{2}(\Omega)\right) \cap L^{2}\left(0, T, \boldsymbol{H}^{1}(\Omega)\right), \\
& p \in L^{2}\left(0, T ; H^{1}(\Omega)\right) .
\end{aligned}
$$

(H1) $\partial \Omega$ is such that the homogeneous Poisson-Neumann problem

$$
-\Delta \phi=g \text { in } \Omega, \quad \frac{\partial \phi}{\partial \boldsymbol{n}}=0 \text { on } \Gamma,
$$

has the regularity property $\|\phi\|_{H^{2}(\Omega)} \leq C\|g\|_{L^{2}(\Omega)}$ for any $g \in L_{0}^{2}(\Omega)$, and the Stokes problem

$$
-\Delta \boldsymbol{v}+\nabla q=\boldsymbol{f} \text { in } \Omega, \quad \nabla \cdot \boldsymbol{v}=0 \quad \text { on } \Omega, \quad \boldsymbol{v}=0 \quad \text { on } \Gamma,
$$

has the regularity property $\|\boldsymbol{v}\|_{H^{2}(\Omega)}+\|q\|_{H^{1}(\Omega)} \leq C\|\boldsymbol{f}\|_{L^{2}(\Omega)}$, for any prescribed $\boldsymbol{f} \in \boldsymbol{L}^{2}(\Omega)$.

(H2) The triangulation of $\Omega$ and the discrete spaces verify:

- Inverse Inequalities:

$$
\begin{aligned}
\left\|\bar{\rho}_{h}\right\|_{L^{3}(\Omega)} \leq C h^{-1 / 2}\left\|\bar{\rho}_{h}\right\|_{L^{2}(\Omega)} & \forall \bar{\rho}_{h} \in W_{h}, \\
\left\|\bar{\rho}_{h}\right\|_{L^{\infty}(\Omega)} \leq C h^{-1 / 2}\left\|\bar{\rho}_{h}\right\|_{H^{1}(\Omega)} & \forall \bar{\rho}_{h} \in W_{h} .
\end{aligned}
$$

- Approximation errors:

$$
\begin{array}{ll}
\inf _{\overline{\boldsymbol{u}}_{h} \in V_{h}}\left\|\overline{\boldsymbol{u}}-\overline{\boldsymbol{u}}_{h}\right\|_{H^{1}(\Omega)} \leq C h\|\overline{\boldsymbol{u}}\|_{H^{2}(\Omega)} & \forall \overline{\boldsymbol{u}} \in \boldsymbol{H}^{2}(\Omega) \cap \boldsymbol{H}_{0}^{1}(\Omega), \\
\inf _{\bar{p}_{h} \in M_{h}}\left\|\bar{p}-\bar{p}_{h}\right\|_{L^{2}(\Omega)} \leq C h\|\bar{p}\|_{H^{1}(\Omega)} & \forall \bar{p} \in H^{1}(\Omega) \cap L_{0}^{2}(\Omega), \\
\inf _{\bar{\rho}_{h} \in W_{h}}\left\|\bar{\rho}-\bar{\rho}_{h}\right\|_{H^{1}(\Omega)} \leq C h\|\bar{\rho}\|_{H^{2}(\Omega)} & \forall \bar{\rho} \in H^{2}(\Omega), \\
\inf _{\bar{\rho}_{h} \in W_{h}}\left\|\bar{\rho}-\bar{\rho}_{h}\right\|_{L^{\infty}(\Omega)} \leq C h^{1 / 2}\|\bar{\rho}\|_{H^{2}(\Omega)} & \forall \bar{\rho} \in H^{2}(\Omega) .
\end{array}
$$

(H3) Inf-sup condition for $\left(\boldsymbol{V}_{h}, M_{h}\right)$. There exists $\beta>0$ (independent of $h$ ) such that

$$
\left\|\bar{p}_{h}\right\|_{L_{0}^{2}(\Omega)} \leq \beta \sup _{\overline{\boldsymbol{u}}_{h} \in V_{h} \backslash\{0\}} \frac{\left(\bar{p}_{h}, \nabla \cdot \overline{\boldsymbol{u}}_{h}\right)}{\left|\nabla \overline{\boldsymbol{u}}_{h}\right|} \forall \bar{p}_{h} \in M_{h} .
$$


(H4) The density and velocity discrete space $\left(\boldsymbol{V}_{h}, W_{h}\right)$ satisfy $\boldsymbol{V}_{h} \cdot \boldsymbol{V}_{h} \subset W_{h}$, that is

$$
\forall \overline{\boldsymbol{u}}_{h}^{1}, \overline{\boldsymbol{u}}_{h}^{2} \in \boldsymbol{V}_{h}, \quad \overline{\boldsymbol{u}}_{h}^{1} \cdot \overline{\boldsymbol{u}}_{h}^{2} \in W_{h}
$$

A manner of defining the discrete spaces $\left(W_{h}, V_{h}, M_{h}\right)$ satisfying hypotheses (H2)(H4) is the following. Let $\left\{\mathcal{T}_{h}\right\}_{h>0}$ be a family of regular, quasi-uniform triangulations of $\Omega$. Then, one can consider $\left(V_{h}, M_{h}\right)$ as the $\left(P_{1}+b_{T}\right) \times P_{1}$ mini-element [7] for the velocity-pressure pair where the bubble function $b_{T}$ is a point-wise linear function that is positive in the interior of each $T \in \mathcal{T}_{h}$ taking the value 1 at the barycenter of $T$ and 0 on $\partial T$. Note that the barycenter of each $T \in \mathcal{T}_{h}$ induces a subdivision of $T$ in three triangles (in $2 D$ ) or four tetrahedrons (in $3 D$ ). Thus, one can approximate the density by the $P_{2}$ finite element on the finer mesh, hence hypothesis $\boldsymbol{V}_{h} \cdot \boldsymbol{V}_{h} \subset W_{h}$ holds.

\subsection{Interpolation operators}

Let $S_{h}^{l}$ be a $P_{l}$-interpolation operator $(l=1,2)$ which is $W^{n, p}(\Omega)$-stable for $1 \leq p \leq$ $\infty$ and $n=0,1$, and inherits the approximation properties from (H2) (for instance, $S_{h}^{l}$ could be the Scott-Zhang or Clement operator [3]). Then, for each $\boldsymbol{v} \in \boldsymbol{L}^{1}(\Omega)$ we define $I_{h} \boldsymbol{v} \in \boldsymbol{V}_{h}$ as follows

$$
\left.I_{h} \boldsymbol{v}\right|_{T}=S_{h}^{1} \boldsymbol{v}+\boldsymbol{c}_{T} b_{T} \quad \boldsymbol{c}_{T}=\frac{1}{\int_{T} b_{T} d \boldsymbol{x}} \int_{T}\left(\boldsymbol{v}-S_{h}^{1} \boldsymbol{v}\right)(\boldsymbol{x}) d \boldsymbol{x}, \quad \forall T \in \mathcal{T}_{h}
$$

where $b_{T}$ is the bubble function defined above. By construction, since $M_{h}$ is defined by $C^{0}$-finite elements locally $P_{1}$, one has

$$
\left(\nabla \cdot\left(\boldsymbol{v}-I_{h} \boldsymbol{v}\right), \bar{p}_{h}\right)=\left(\boldsymbol{v}-I_{h} \boldsymbol{v}, \nabla \bar{p}_{h}\right)=0 \quad \forall \boldsymbol{v} \in \boldsymbol{H}_{0}^{1}(\Omega) \text { and } \bar{p}_{h} \in M_{h} .
$$

On the other hand, for each $p \in H^{1}(\Omega)$ and $\rho \in L^{1}(\Omega)$ we consider $J_{h} p=S_{h}^{1} p \in$ $M_{h}$ and $K_{h} \rho=S_{h}^{2} \rho \in W_{h}$ as interpolation operators related to pressure and density respectively ( $K_{h}$ acts over the finer mesh). Let us sum up the properties to be needed for the interpolation operators $I_{h}, J_{h}$, and $K_{h}$ in the following.

Lemma 4 Suppose that hypotheses $(H 1)-(H 3)$ hold. Then, for any $n=0,1$ and $1 \leq p \leq+\infty$, one has

$$
\begin{aligned}
\left\|I_{h} \boldsymbol{v}\right\|_{W^{n, p}(\Omega)} & \leq C\|\boldsymbol{v}\|_{W^{n, p}(\Omega)}, \quad\left\|I_{h} \boldsymbol{v}-\boldsymbol{v}\right\|_{W^{n, p}} \leq C h|\boldsymbol{v}|_{W^{n+1, p}(\Omega)}, \\
\left\|K_{h} \rho\right\|_{W^{n, p}(\Omega)} & \leq C\|\rho\|_{W^{n, p}(\Omega)}, \quad\left\|K_{h} \rho-\rho\right\|_{W^{n, p}} \leq C h|\rho|_{W^{n+1, p}(\Omega)}, \\
\left\|K_{h} \rho-\rho\right\|_{L^{\infty}} & \leq C h^{1 / 2}\|\rho\|_{H^{2}(\Omega)} \\
\left\|J_{h} p-p\right\|_{L^{2}(\Omega)} & \leq C h\|p\|_{H^{1}(\Omega)} .
\end{aligned}
$$




\section{Point-wise estimates for the discrete density}

We give here the proof of point-wise stability estimates for the discrete density (given in (26)) under hypothesis $(S)$. The idea to prove this type of estimates has already been used in [10], by means of a truncation operator, the estimate $k \sum_{l=0}^{N-1}\left|\nabla \boldsymbol{u}_{h}^{l}\right|^{2} \leq C_{s}$, and the $H^{1}$-projection of $\boldsymbol{u}_{h}^{n}$ onto a finite-element space of higher order in the discrete density equation (9). If we now admit a better bound for the discrete velocity, namely $\max _{l=1, \ldots, N-1}\left|\nabla \boldsymbol{u}_{h}^{l}\right| \leq C_{s}$, discrete maximum principle holds without the projection of the discrete velocity acting in (9). Such a estimate will be a consequence of the error estimates in weak norms for the velocity [see (51)].

The proof of such a maximum principle will be divided into three steps: corresponding to Lemmas 5, 6, and Corollary 7.

Consider the following auxiliary time-stepping scheme: Let $\rho^{0}=\rho_{0}$. Given $\rho^{n} \in$ $H^{1}(\Omega)$, find $\rho^{n+1} \in H^{2}(\Omega)$ as the solution to the elliptic problem

$$
\frac{\rho^{n+1}-\rho^{n}}{k}+\boldsymbol{u}_{h}^{n} \cdot \nabla \rho^{n+1}-\lambda \Delta \rho^{n+1}=0 \text { in } \Omega,\left.\quad \frac{\partial \rho^{n+1}}{\partial \boldsymbol{n}}\right|_{\partial \Omega}=0 .
$$

This problem is well-posed thanks to the elliptic regularity imposed in $(H 1)$.

The proof of the following result was done in [10] in Appendix (A.1).

Lemma 5 Fix $n=0, \ldots, N-1$. Suppose that there exists a unique solution $\left(\boldsymbol{u}_{h}^{l}, p_{h}^{l}\right)$ of (9)-(11) for $l=1, \ldots, n$ satisfying (jointly to initialization $\boldsymbol{u}_{h}^{0}$ ) the estimate

$$
\left|\nabla \boldsymbol{u}_{h}^{l}\right| \leq C_{s} \quad \forall l=0, \ldots, n
$$

with $C_{s}>0$ being a constant independent of $(k, h)$, and steps $l=1, \ldots, n$. Then, for $k$ small enough (depending only on $C_{s}$ ), we have

$$
\begin{aligned}
& 0<m \leq \rho^{l+1} \leq M, \quad \forall l=0, \ldots, n . \\
& \max _{0 \leq l \leq n}\left\|\rho^{l+1}\right\|_{H^{1}(\Omega)}^{2} \leq C, \quad k \sum_{l=0}^{n}\left\|\rho^{l+1}\right\|_{H^{2}(\Omega)}^{2} \leq C,
\end{aligned}
$$

where $C$ is a constant independent of $(h, k)$, and steps $l=1, \ldots, n$.

The next lemma provides a comparison between problems (9) and (21).

Lemma 6 Fix $n=0, \ldots, N-1$. Under assumptions of Lemma 5, the discrete density $\left(\rho_{h}^{l}\right)_{l=1}^{n+1}$ of scheme (9) satisfies, for $h$ and $k$ small enough (independent of the time step $n)$ :

$$
k \sum_{l=0}^{n}\left\|\rho_{h}^{l+1}-K_{h} \rho^{l+1}\right\|_{H^{1}(\Omega)}^{2} \leq C h^{2}
$$

where $C>0$ is a constant independent of $(h, k)$ and steps $l=1, \ldots, n$. 
The details of the proof of Lemma 6 are similar to that of Theorem 2 in most arguments. Since we compare (9) with a time-stepping scheme being linear on $\rho^{n+1}$ for a fixed data $\boldsymbol{u}_{h}^{n}$, no consistency errors must be bounded. Therefore, the proof of (25) is slightly shorter than the proof of error estimates given in Theorem 2, and therefore the proof is omitted.

Corollary 7 Fix $n=0, \ldots, N-1$. Under the assumption of Lemma 6, the discrete density of scheme (9) satisfies, for $h$ and $k$ small enough (depending only on $C_{s}$ ):

$$
0<\tilde{m} \leq \rho_{h}^{l+1} \leq \tilde{M}, \quad \forall l: 0 \leq l \leq n
$$

where $\widetilde{m}$ and $\widetilde{M}$ are the constants fixed in (12).

Proof It follows easily from (25) and inverse inequality (18) that $\| \rho_{h}^{l+1}-K_{h}$ $\rho^{l+1} \|_{L^{\infty}(\Omega)}^{2} \leq C h / k$. This estimate jointly to approximation error (20) for $\bar{\rho}=\rho^{l+1}$ and (25) imply that

$$
\left\|\rho_{h}^{l+1}-\rho^{l+1}\right\|_{L^{\infty}(\Omega)}^{2} \leq C h / k
$$

Indeed,

$k \sum_{l}\left\|\rho_{h}^{l+1}-\rho^{l+1}\right\|_{L^{\infty}}^{2} \leq C k \sum_{l}\left(\left\|\rho_{h}^{l+1}-K_{h} \rho^{l+1}\right\|_{L^{\infty}}^{2}+\left\|K_{h} \rho^{l+1}-\rho^{l+1}\right\|_{L^{\infty}}^{2}\right)$,

hence by using firstly (18) and (20) and secondly (24) and (25):

$$
k \sum_{l}\left\|\rho_{h}^{l+1}-\rho^{l+1}\right\|_{L^{\infty}}^{2} \leq \frac{C}{h} k \sum_{l}\left\|\rho_{h}^{l+1}-K_{h} \rho^{l+1}\right\|_{H^{1}}^{2}+C h k \sum_{l}\left\|\rho^{l+1}\right\|_{H^{2}}^{2} \leq C h
$$

one arrives at (27).

Finally, note that, for $h$ and $k$ small enough (independent of the time step $n$ ), (26) holds from constraint (S), (23) and (27).

\section{Well-posedness of scheme (9)-(11) and weak error estimates}

First of all, we are going to introduce the consistency errors and the error equations for both velocity and density. To motivate the way consistency errors will be written below, let us indicate what we would obtain if a usual methodology of writing them were used. Typically, the consistency error are obtained by putting the differential solution related to (8) into the numerical scheme (9)-(11). In doing so, one arrives at

$$
\begin{gathered}
\frac{\rho\left(t_{n+1}\right)-\rho\left(t_{n}\right)}{k}+\boldsymbol{u}\left(t_{n}\right) \cdot \nabla \rho\left(t_{n+1}\right)-\lambda \Delta \rho\left(t_{n+1}\right)=R_{\rho}^{n+1}, \\
\left\{\begin{array}{l}
\rho\left(t_{n}\right) \frac{\boldsymbol{u}\left(t_{n+1}\right)-\boldsymbol{u}\left(t_{n}\right)}{k}+\left(\rho\left(t_{n+1}\right) \boldsymbol{u}\left(t_{n}\right)-\lambda \nabla \rho\left(t_{n+1}\right)\right) \cdot \nabla \boldsymbol{u}\left(t_{n+1}\right) \\
-\nabla \cdot\left(\mu \nabla \boldsymbol{u}\left(t_{n+1}\right)-\lambda \rho\left(t_{n+1}\right)\left(\nabla \boldsymbol{u}\left(t_{n+1}\right)\right)^{t}\right)+\nabla p\left(t_{n+1}\right)=\rho\left(t_{n+1}\right) \boldsymbol{f}\left(t_{n+1}\right)+R_{\boldsymbol{u}}^{n+1},
\end{array}\right.
\end{gathered}
$$


where $R_{\rho}^{n+1}$ and $R_{u}^{n+1}$ are the time consistency errors having the expressions

$$
\begin{aligned}
R_{\rho}^{n+1}= & \frac{1}{k} \int_{t_{n}}^{t_{n+1}}\left(s-t_{n}\right) \rho_{t t} d s-\left(\int_{t_{n}}^{t_{n+1}} \boldsymbol{u}_{t}(s) d s\right) \cdot \nabla \rho\left(t_{n+1}\right), \\
R_{\boldsymbol{u}}^{n+1}= & \frac{\rho\left(t_{n+1}\right)}{k} \int_{t_{n}}^{t_{n+1}}\left(s-t_{n}\right) \boldsymbol{u}_{t t} d s-\frac{1}{k}\left(\int_{t_{n}}^{t_{n+1}} \rho_{t}(s) d s\right)\left(\int_{t_{n}}^{t_{n+1}} \boldsymbol{u}_{t}(s) d s\right) \\
& -\rho\left(t_{n+1}\right)\left(\int_{t_{n}}^{t_{n+1}} \boldsymbol{u}_{t}(s) d s\right) \cdot \nabla \boldsymbol{u}\left(t_{n+1}\right) .
\end{aligned}
$$

Obviously, writing the exact solution in the above context requires that some sort of regularity for $\boldsymbol{u}_{t t}$ must be imposed. It is easy to realize that at some moment in obtaining the error estimates, by using this approach, we must bound the term where $\boldsymbol{u}_{t t}$ appears in $R_{\boldsymbol{u}}^{n+1}$ acting on $\overline{\boldsymbol{u}}_{h} \in \boldsymbol{V}_{h}$ :

$$
\begin{aligned}
& k\left(\frac{\rho\left(t_{n+1}\right)}{k} \int_{t_{n}}^{t_{n+1}}\left(s-t_{n}\right) \boldsymbol{u}_{t t} d s, \overline{\boldsymbol{u}}_{h}\right) \\
& \quad \leq\left\|\rho\left(t_{n+1}\right)\right\|_{L^{\infty}(\Omega)} \int_{t_{n}}^{t_{n+1}}\left(s-t_{n}\right)\left\|\boldsymbol{u}_{t t}(s)\right\|_{L^{6 / 5}(\Omega)}\left\|\overline{\boldsymbol{u}}_{h}\right\|_{L^{6}(\Omega)} \\
& \quad \leq C \frac{1}{k}\left(\int_{t_{n}}^{t_{n+1}}\left(s-t_{n}\right)\left\|\boldsymbol{u}_{t t}(s)\right\|_{L^{6 / 5}(\Omega)}\right)^{2}+\varepsilon k\left|\nabla \overline{\boldsymbol{u}}_{h}\right|^{2}
\end{aligned}
$$

The best regularity for $\boldsymbol{u}_{t t}$ we can expect (without imposing global compatibility conditions) is $\sigma^{1 / 2} \boldsymbol{u}_{t t} \in L^{2}\left(0, T ; \boldsymbol{L}^{6 / 5}(\Omega)\right)$, where $\sigma(t)=\min \{1, t\}$. Therefore,

$k\left(\frac{\rho\left(t_{n+1}\right)}{k} \int_{t_{n}}^{t_{n+1}}\left(s-t_{n}\right) \boldsymbol{u}_{t t} d s, \overline{\boldsymbol{u}}_{h}\right) \leq C k \int_{t_{n}}^{t_{n+1}}\left(s-t_{n}\right)\left\|\boldsymbol{u}_{t t}(s)\right\|_{L^{6 / 5}(\Omega)}^{2}+\varepsilon k\left|\nabla \overline{\boldsymbol{u}}_{h}\right|^{2}$,

but it is easy to check that this bound only implies the suboptimal error estimates in time $\mathcal{O}\left(k^{1 / 2}\right)$. In order for the result of $\mathcal{O}(k)$ to hold, the regularity condition $\boldsymbol{u}_{t t} \in L^{2}\left(0, T ; \boldsymbol{L}^{6 / 5}(\Omega)\right)$ must be imposed, but it requires a nonlocal compatibility condition for the pressure at the initial time $t=0$, depending on the data $\boldsymbol{u}_{0}, \rho_{0}$ and $\boldsymbol{f}(0)$, which cannot be verified in practice [12]. Therefore, it is clear that we must write the consistency errors in which $\boldsymbol{u}_{t t}$ not to appear.

It is well at this point to point out that in the particular case of constant density (that is, for the classical Navier-Stokes problem), to obtain the optimal order $\mathcal{O}(k)$, 
it suffices to impose $\boldsymbol{u}_{t t} \in L^{2}\left(0, T ; \boldsymbol{V}^{\prime}\right)$, which is a regularity hypothesis that not requires the above-mentioned nonlocal compatibility condition.

Throughout the paper we will denote the errors as follows:

$$
\begin{aligned}
\boldsymbol{e}_{\boldsymbol{u}}^{n} & =\boldsymbol{e}_{d, \boldsymbol{u}}+\boldsymbol{e}_{i, \boldsymbol{u}}, \text { with } \boldsymbol{e}_{d, \boldsymbol{u}}=\boldsymbol{u}_{h}^{n}-I_{h} \boldsymbol{u}\left(t_{n}\right) \quad \text { and } \quad \boldsymbol{e}_{i, \boldsymbol{u}}=I_{h} \boldsymbol{u}\left(t_{n}\right)-\boldsymbol{u}\left(t_{n}\right), \\
e_{p}^{n} & =e_{d, p}+e_{i, p}, \text { with } e_{d, p}=p_{h}^{n}-J_{h} p\left(t_{n}\right) \quad \text { and } \quad e_{i, p}=J_{h} p\left(t_{n}\right)-p\left(t_{n}\right), \\
e_{\rho}^{n} & =e_{d, \rho}+e_{i, \rho}, \text { with } e_{d, \rho}=\rho_{h}^{n}-K_{h} \rho\left(t_{n}\right) \quad \text { and } \quad e_{i, \rho}=K_{h} \rho\left(t_{n}\right)-\rho\left(t_{n}\right),
\end{aligned}
$$

where $e_{i}$, represents the error coming from the interpolation error, and $e_{d}$, represents the error related to the nonlinearity in problem (2) (discrete error).

\subsection{Error equation for the velocity-pressure}

To introduce a new consistency error let us first begin by integrating $(8)_{1}$ with respect to time between $t_{n}$ and $t_{n+1}$ to get

$$
\begin{aligned}
& \int_{t_{n}}^{t_{n+1}}\left(\rho(s) \boldsymbol{u}_{t}(s), \overline{\boldsymbol{u}}\right) d s+\int_{t_{n}}^{t_{n+1}}(((\rho(s) \boldsymbol{u}(s)-\lambda \nabla \rho(s)) \cdot \nabla) \boldsymbol{u}(s), \overline{\boldsymbol{u}}) d s \\
& \quad+\int_{t_{n}}^{t_{n+1}}\left(\mu \nabla \boldsymbol{u}(s)-\lambda \rho(s)(\nabla \boldsymbol{u}(s))^{t}, \nabla \overline{\boldsymbol{u}}\right) d s-\int_{t_{n}}^{t_{n+1}}(p(s), \nabla \cdot \overline{\boldsymbol{u}}) d s \\
& =\int_{t_{n}}^{t_{n+1}}(\rho(s) \boldsymbol{f}(s), \overline{\boldsymbol{u}}) d s
\end{aligned}
$$

for all $\overline{\boldsymbol{u}} \in \boldsymbol{H}_{0}^{1}(\Omega)$. If follows easily that the integral of the time derivative may be written as

$$
\begin{aligned}
\int_{t_{n}}^{t_{n+1}}\left(\rho \boldsymbol{u}_{t}(s), \overline{\boldsymbol{u}}\right) d s & =k\left(\rho\left(t_{n}\right) \delta_{t} \boldsymbol{u}\left(t_{n+1}\right), \overline{\boldsymbol{u}}\right)+\int_{t_{n}}^{t_{n+1}}\left(\left[\rho(s)-\rho\left(t_{n}\right)\right] \boldsymbol{u}_{t}(s), \overline{\boldsymbol{u}}\right) d s \\
& :=k\left(\rho\left(t_{n}\right) \delta_{t} \boldsymbol{u}\left(t_{n+1}\right), \overline{\boldsymbol{u}}\right)+\left(\xi_{\boldsymbol{u}, 1}^{n+1}, \overline{\boldsymbol{u}}\right) .
\end{aligned}
$$

This expression will allow us to compare the time derivative terms corresponding to (10) and (28) in a suitable way. By subtracting (28), particularized for $\overline{\boldsymbol{u}}=\overline{\boldsymbol{u}}_{h} \in \boldsymbol{V}_{h}$, from (10), we find the relation

$$
\begin{aligned}
& k\left(\rho_{h}^{n} \delta_{t} \boldsymbol{u}_{h}^{n+1}-\rho\left(t_{n}\right) \delta_{t} \boldsymbol{u}\left(t_{n+1}\right), \overline{\boldsymbol{u}}_{h}\right)-\left(\xi_{\boldsymbol{u}, 1}^{n+1}, \overline{\boldsymbol{u}}_{h}\right) \\
& \quad+\int_{t_{n}}^{t_{n+1}}\left(\left(\rho_{h}^{n+1} \boldsymbol{u}_{h}^{n} \cdot \nabla\right) \boldsymbol{u}_{h}^{n+1}-(\rho(s) \boldsymbol{u}(s) \cdot \nabla) \boldsymbol{u}(s), \overline{\boldsymbol{u}}_{h}\right) d s
\end{aligned}
$$




$$
\begin{aligned}
& -\lambda \int_{t_{n}}^{t_{n+1}}\left(\left(\nabla \rho_{h}^{n+1} \cdot \nabla\right) \boldsymbol{u}_{h}^{n+1}-(\nabla \rho(s) \cdot \nabla) \rho(s), \overline{\boldsymbol{u}}_{h}\right) d s \\
& +\mu \int_{t_{n}}^{t_{n+1}}\left(\nabla \boldsymbol{u}_{h}^{n+1}-\nabla \boldsymbol{u}(s), \overline{\boldsymbol{u}}_{h}\right) d s \\
& -\lambda \int_{t_{n}}^{t_{n+1}}\left(\left(\rho_{h}^{n+1}-\rho_{\tilde{m}}^{\widetilde{M}}\right)\left(\nabla \boldsymbol{u}_{h}^{n+1}\right)^{t}-\rho(s)(\nabla \boldsymbol{u}(s))^{t}, \nabla \overline{\boldsymbol{u}}_{h}\right) d s \\
& +\frac{1}{2} \int_{t_{n}}^{t_{n+1}}\left(\nabla \cdot \boldsymbol{u}_{h}^{n} \rho_{h}^{n+1}, \boldsymbol{u}_{h}^{n+1} \cdot \overline{\boldsymbol{u}}_{h}\right) d s-\int_{t_{n}}^{t_{n+1}}\left(p_{h}^{n+1}-p(s), \nabla \cdot \overline{\boldsymbol{u}}_{h}\right) d s \\
& -\int_{t_{n}}^{t_{n+1}}\left(\rho_{h}^{n+1} \boldsymbol{f}\left(t_{n+1}\right)-\rho(s) \boldsymbol{f}(s), \overline{\boldsymbol{u}}_{h}\right) d s=0 .
\end{aligned}
$$

We now split each pair of terms in the above equation in preparation for deriving the error estimates for the discrete velocity error, $\boldsymbol{e}_{d, \boldsymbol{u}}^{n+1}=\boldsymbol{u}_{h}^{n+1}-I_{h} \boldsymbol{u}\left(t_{n+1}\right)$. To begin with, we treat the time derivative terms in (29) as

$$
\begin{aligned}
& k\left(\rho_{h}^{n} \delta_{t} \boldsymbol{u}_{h}^{n+1}-\rho\left(t_{n}\right) \delta_{t} \boldsymbol{u}\left(t_{n+1}\right), \overline{\boldsymbol{u}}_{h}\right)=k\left(\rho_{h}^{n} \delta_{t} \boldsymbol{e}_{d, \boldsymbol{u}}^{n+1}, \overline{\boldsymbol{u}}_{h}\right)+k\left(\rho_{h}^{n} \delta_{t} \boldsymbol{e}_{i, \boldsymbol{u}}^{n+1}, \overline{\boldsymbol{u}}_{h}\right) \\
& \quad+k\left(e_{d, \rho}^{n} \delta_{t} \boldsymbol{u}\left(t_{n+1}\right), \overline{\boldsymbol{u}}_{h}\right)+k\left(e_{i, \rho}^{n} \delta_{t} \boldsymbol{u}\left(t_{n+1}\right), \overline{\boldsymbol{u}}_{h}\right) \\
& \quad:=k\left(\rho_{h}^{n} \delta_{t} \boldsymbol{e}_{d, \boldsymbol{u}}^{n+1}, \overline{\boldsymbol{u}}_{h}\right)+\sum_{j=2}^{4}\left(\xi_{\boldsymbol{u}, j}^{n+1}, \overline{\boldsymbol{u}}_{h}\right) .
\end{aligned}
$$

For the convective term in (29) we propose the following decomposition:

$$
\begin{aligned}
& \int_{t_{n}}^{t_{n+1}}\left(\left(\rho_{h}^{n+1} \boldsymbol{u}_{h}^{n} \cdot \nabla\right) \boldsymbol{u}_{h}^{n+1}-(\rho(s) \boldsymbol{u}(s) \cdot \nabla) \boldsymbol{u}(s), \overline{\boldsymbol{u}}_{h}\right) d s \\
& =k\left(\left(\rho_{h}^{n+1} \boldsymbol{u}_{h}^{n} \cdot \nabla\right) \boldsymbol{e}_{d, \boldsymbol{u}}^{n+1}, \overline{\boldsymbol{u}}_{h}\right)+k\left(\left(\left(\rho_{h}^{n+1} \boldsymbol{e}_{d, \boldsymbol{u}}^{n}+e_{d, \rho}^{n+1} I_{h} \boldsymbol{u}\left(t_{n}\right) \cdot \nabla\right) I_{h} \boldsymbol{u}\left(t_{n+1}\right), \overline{\boldsymbol{u}}_{h}\right)\right. \\
& \quad+\int_{t_{n}}^{t_{n+1}}\left(\left(K_{h} \rho\left(t_{n+1}\right)\left[I_{h} \boldsymbol{u}\left(t_{n}\right)-I_{h} \boldsymbol{u}(s)\right] \cdot \nabla\right) I_{h} \boldsymbol{u}\left(t_{n+1}\right), \overline{\boldsymbol{u}}_{h}\right) d s \\
& \quad+\int_{t_{n}}^{t_{n+1}}\left(\left(\left[K_{h} \rho\left(t_{n+1}\right)-K_{h} \rho(s)\right] I_{h} \boldsymbol{u}(s) \cdot \nabla\right) I_{h} \boldsymbol{u}\left(t_{n+1}\right), \overline{\boldsymbol{u}}_{h}\right) d s
\end{aligned}
$$




$$
\begin{aligned}
& +\int_{t_{n}}^{t_{n+1}}\left(\left(K_{h} \rho(s)\left[I_{h} \boldsymbol{u}(s)-\boldsymbol{u}(s)\right] \cdot \nabla\right) I_{h} \boldsymbol{u}\left(t_{n+1}\right), \overline{\boldsymbol{u}}_{h}\right) d s \\
& +\int_{t_{n}}^{t_{n+1}}\left(\left(\left[K_{h} \rho(s)-\rho(s)\right] \boldsymbol{u}(s) \cdot \nabla\right) I_{h} \boldsymbol{u}\left(t_{n+1}\right), \overline{\boldsymbol{u}}_{h}\right) d s \\
& +\int_{t_{n}}^{t_{n+1}}\left((\rho(s) \boldsymbol{u}(s) \cdot \nabla)\left[I_{h} \boldsymbol{u}\left(t_{n+1}\right)-I_{h} \boldsymbol{u}(s)\right], \overline{\boldsymbol{u}}_{h}\right) d s \\
& +\int_{t_{n}}^{t_{n+1}}\left((\rho(s) \boldsymbol{u}(s) \cdot \nabla)\left[I_{h} \boldsymbol{u}(s)-\boldsymbol{u}(s)\right], \overline{\boldsymbol{u}}_{h}\right) d s \\
& :=k\left(\left(\rho_{h}^{n+1} \boldsymbol{u}_{h}^{n} \cdot \nabla\right) \boldsymbol{e}_{d, \boldsymbol{u}}^{n+1}, \overline{\boldsymbol{u}}_{h}\right)+\left(\zeta_{\boldsymbol{u}, 1}^{n+1}, \overline{\boldsymbol{u}}_{h}\right)+\sum_{j=5}^{10}\left(\xi_{\boldsymbol{u}, j}^{n+1}, \overline{\boldsymbol{u}}_{h}\right) .
\end{aligned}
$$

The first $\lambda$-term in (29) may be written as follows

$$
\begin{aligned}
-\lambda \int_{t_{n}}^{t_{n+1}}\left(\left(\nabla \rho_{h}^{n+1} \cdot \nabla\right) \boldsymbol{u}_{h}^{n+1}-(\nabla \rho(s) \cdot \nabla) \rho(s), \overline{\boldsymbol{u}}_{h}\right) d s \\
=-\lambda k\left(\left(\nabla \rho_{h}^{n+1} \cdot \nabla\right) \boldsymbol{e}_{d, \boldsymbol{u}}^{n+1}, \overline{\boldsymbol{u}}_{h}\right)-\lambda k\left(\left(\nabla e_{d, \rho}^{n+1} \cdot \nabla\right) I_{h} \boldsymbol{u}\left(t_{n+1}\right), \overline{\boldsymbol{u}}_{h}\right) \\
-\lambda \int_{t_{n}}^{t_{n+1}}\left(\left(\left[\nabla K_{h} \rho\left(t_{n+1}\right)-\nabla K_{h} \rho(s)\right] \cdot \nabla\right) I_{h} \boldsymbol{u}\left(t_{n+1}\right), \overline{\boldsymbol{u}}_{h}\right) d s \\
-\lambda \int_{t_{n}}^{t_{n+1}}\left(\left(\left[\nabla K_{h} \rho(s)-\nabla \rho(s)\right] \cdot \nabla\right) I_{h} \boldsymbol{u}\left(t_{n+1}\right), \overline{\boldsymbol{u}}_{h}\right) d s \\
-\lambda \int_{t_{n}}^{t_{n+1}}\left((\nabla \rho(s) \cdot \nabla)\left[I_{h} \boldsymbol{u}\left(t_{n+1}\right)-I_{h} \boldsymbol{u}(s)\right], \overline{\boldsymbol{u}}_{h}\right) d s \\
-\lambda \int_{t_{n}}^{t_{n+1}}\left((\nabla \rho(s) \cdot \nabla)\left[I_{h} \boldsymbol{u}(s)-\boldsymbol{u}(s)\right], \overline{\boldsymbol{u}}_{h}\right) d s \\
:=-\lambda k\left(\left(\nabla \rho_{h}^{n+1} \cdot \nabla\right) \boldsymbol{e}_{d, \boldsymbol{u}}^{n+1}, \overline{\boldsymbol{u}}_{h}\right)+\left(\zeta_{\boldsymbol{u}, 2}^{n+1}, \overline{\boldsymbol{u}}_{h}\right)+\sum_{j=11}^{14}\left(\xi_{\boldsymbol{u}, j}^{n+1}, \overline{\boldsymbol{u}}_{h}\right) .
\end{aligned}
$$


Concerning the diffusion term in (29) we partition it as

$$
\begin{aligned}
\mu \int_{t_{n}}^{t_{n+1}}\left(\nabla \boldsymbol{u}_{h}^{n+1}-\nabla \boldsymbol{u}(s), \nabla \overline{\boldsymbol{u}}_{h}\right) d s= & \mu k\left(\nabla \boldsymbol{e}_{d, \boldsymbol{u}}^{n+1}, \nabla \overline{\boldsymbol{u}}_{h}\right) \\
& +\mu \int_{t_{n}}^{t_{n+1}}\left(\nabla I_{h} \boldsymbol{u}\left(t_{n+1}\right)-\nabla I_{h} \boldsymbol{u}(s), \nabla \overline{\boldsymbol{u}}_{h}\right) d s \\
& +\mu \int_{t_{n}}^{t_{n+1}}\left(\nabla I_{h} \boldsymbol{u}(s)-\nabla \boldsymbol{u}(s), \nabla \overline{\boldsymbol{u}}_{h}\right) d s \\
:= & \mu k\left(\nabla \boldsymbol{e}_{d, \boldsymbol{u}}^{n+1}, \nabla \overline{\boldsymbol{u}}_{h}\right)+\sum_{j=15}^{16}\left(\xi_{\boldsymbol{u}, j}^{n+1}, \overline{\boldsymbol{u}}_{h}\right) .
\end{aligned}
$$

Observe that $\int_{\Omega}(\nabla \boldsymbol{u}(s))^{t}: \nabla \boldsymbol{u}(s) d \boldsymbol{x}=0$, which is true owing to $\nabla \cdot \boldsymbol{u}(s)=0$ in $\Omega$ and $\boldsymbol{u}(s)=0$ on $\Gamma$. Then the other $\lambda$-term in (29) may be written as

$$
\begin{aligned}
&-\lambda \int_{t_{n}}^{t_{n+1}}\left(\left(\rho_{h}^{n+1}-\rho_{m}^{M}\right)\left(\nabla \boldsymbol{u}_{h}^{n+1}\right)^{t}-\left(\rho(s)-\rho_{\widetilde{m}}^{\widetilde{M}}\right)(\nabla \boldsymbol{u}(s))^{t}, \nabla \overline{\boldsymbol{u}}_{h}\right) d s \\
&=-\lambda k\left(\left(\rho_{h}^{n+1}-\rho_{\tilde{m}}^{\widetilde{M}}\right)\left(\nabla \boldsymbol{e}_{d, \boldsymbol{u}}^{n+1}\right)^{t}, \nabla \overline{\boldsymbol{u}}_{h}\right)-\lambda k\left(e_{d, \rho}^{n+1}\left(\nabla I_{h} \boldsymbol{u}\left(t_{n+1}\right)\right)^{t}, \nabla \overline{\boldsymbol{u}}_{h}\right) \\
&-\lambda \int_{t_{n}}^{t_{n+1}}\left(\left[K_{h} \rho\left(t_{n+1}\right)-K_{h} \rho(s)\right]\left(\nabla I_{h} \boldsymbol{u}\left(t_{n+1}\right)\right)^{t}, \nabla \overline{\boldsymbol{u}}_{h}\right) d s \\
&-\lambda \int_{t_{n}}^{t_{n+1}}\left(\left[K_{h} \rho(s)-\rho(s)\right]\left(\nabla I_{h} \boldsymbol{u}\left(t_{n+1}\right)\right)^{t}, \nabla \overline{\boldsymbol{u}}_{h}\right) d s \\
&-\lambda \int_{t_{n}}^{t_{n+1}}\left(\left(\rho(s)-\rho_{\widetilde{m}}^{\widetilde{M}}\right)\left[\nabla I_{h} \boldsymbol{u}\left(t_{n+1}\right)-\nabla I_{h} \boldsymbol{u}(s)\right]^{t}, \nabla \overline{\boldsymbol{u}}_{h}\right) d s \\
&-\lambda \int_{t_{n}}^{t_{n+1}}\left(\left(\rho(s)-\rho_{\tilde{m}}^{\widetilde{M}}\right)\left[\nabla I_{h} \boldsymbol{u}(s)-\nabla \boldsymbol{u}(s)\right]^{t}, \nabla \overline{\boldsymbol{u}}_{h}\right) d s \\
&=-\lambda k\left(\left(\rho_{h}^{n+1}-\rho_{\widetilde{m}}^{\widetilde{M}}\right)\left(\nabla \boldsymbol{e}_{d, \boldsymbol{u}}^{n+1}\right)^{t}, \nabla \overline{\boldsymbol{u}}_{h}\right)+\left(\zeta_{\boldsymbol{u}, 3}^{n+1}, \overline{\boldsymbol{u}}_{h}\right)+\sum_{j=17}^{20}\left(\xi_{u, j}^{n+1}, \overline{\boldsymbol{u}}_{h}\right) .
\end{aligned}
$$


An similar argument to the convective and diffusion term shows that the stabilizing term of (10) takes the form

$$
\begin{aligned}
& \frac{k}{2}\left(\nabla \cdot \boldsymbol{u}_{h}^{n} \rho_{h}^{n+1}, \boldsymbol{u}_{h}^{n+1} \cdot \overline{\boldsymbol{u}}_{h}\right)=\frac{k}{2}\left(\nabla \cdot \boldsymbol{u}_{h}^{n} \rho_{h}^{n+1}, \boldsymbol{e}_{d, \boldsymbol{u}}^{n+1} \cdot \overline{\boldsymbol{u}}_{h}\right) \\
& \quad+\frac{k}{2}\left(\nabla \cdot \boldsymbol{e}_{d, \boldsymbol{u}}^{n} \rho_{h}^{n+1}, I_{h} \boldsymbol{u}\left(t_{n+1}\right) \cdot \overline{\boldsymbol{u}}_{h}\right) \\
& \quad+\frac{1}{2} \int_{t_{n}}^{t_{n+1}}\left(\nabla \cdot\left[I_{h} \boldsymbol{u}\left(t_{n}\right)-I_{h} \boldsymbol{u}(s)\right] \rho_{h}^{n+1}, I_{h} \boldsymbol{u}\left(t_{n+1}\right) \cdot \overline{\boldsymbol{u}}_{h}\right) d s \\
& \quad+\frac{1}{2} \int_{t_{n}}^{t_{n+1}}\left(\nabla \cdot\left[I_{h} \boldsymbol{u}(s)-\boldsymbol{u}(s)\right] \rho_{h}^{n+1}, I_{h} \boldsymbol{u}\left(t_{n+1}\right) \cdot \overline{\boldsymbol{u}}_{h}\right) d s \\
& :=\frac{k}{2}\left(\nabla \cdot \boldsymbol{u}_{h}^{n} \rho_{h}^{n+1}, \boldsymbol{e}_{d, \boldsymbol{u}}^{n+1} \cdot \overline{\boldsymbol{u}}_{h}\right)+\left(\zeta_{\boldsymbol{u}, 4}^{n+1}, \overline{\boldsymbol{u}}_{h}\right)+\sum_{j=21}^{22}\left(\xi_{\boldsymbol{u}, j}^{n+1}, \overline{\boldsymbol{u}}_{h}\right),
\end{aligned}
$$

and the pressure terms become

$$
\begin{aligned}
-\int_{t_{n}}^{t_{n+1}}\left(p_{h}^{n+1}-p(s), \nabla \cdot \overline{\boldsymbol{u}}_{h}\right) d s= & -k\left(p_{h}^{n+1}-J_{h} p\left(t_{n+1}\right), \nabla \cdot \overline{\boldsymbol{u}}_{h}\right) \\
& -\int_{t_{n}}^{t_{n+1}}\left(J_{h} p\left(t_{n+1}\right)-J_{h} p(s), \nabla \cdot \overline{\boldsymbol{u}}_{h}\right) d s \\
& -\int_{t_{n}}^{t_{n+1}}\left(J_{h} p(s)-p(s), \nabla \cdot \overline{\boldsymbol{u}}_{h}\right) d s \\
:= & -k\left(e_{d, p}^{n+1}, \nabla \cdot \overline{\boldsymbol{u}}_{h}\right)+\sum_{j=23}^{24}\left(\xi_{\boldsymbol{u}, j}^{n+1}, \overline{\boldsymbol{u}}_{h}\right) .
\end{aligned}
$$

To conclude we handle the forcing term that unlike the Navier-Stokes equation provides extra consistency terms to be bounded. Thus we have

$$
\begin{aligned}
& -\int_{t_{n}}^{t_{n+1}}\left(\rho_{h}^{n+1} \boldsymbol{f}\left(t_{n+1}\right)-\rho(s) \boldsymbol{f}(s), \overline{\boldsymbol{u}}_{h}\right) d s=-k\left(e_{d, \rho}^{n+1} \boldsymbol{f}\left(t_{n+1}\right), \overline{\boldsymbol{u}}_{h}\right) \\
& -\int_{t_{n}}^{t_{n+1}}\left(\left[K_{h} \rho\left(t_{n+1}\right)-K_{h} \rho(s)\right] \boldsymbol{f}\left(t_{n+1}\right), \overline{\boldsymbol{u}}_{h}\right) d s
\end{aligned}
$$




$$
\begin{aligned}
& -\int_{t_{n}}^{t_{n+1}}\left(\left[K_{h} \rho(s)-\rho(s)\right] \boldsymbol{f}\left(t_{n+1}\right), \overline{\boldsymbol{u}}_{h}\right) d s \\
& -\int_{t_{n}}^{t_{n+1}}\left(\rho(s)\left[\boldsymbol{f}\left(t_{n+1}\right)-\boldsymbol{f}(s)\right], \overline{\boldsymbol{u}}_{h}\right) d s \\
& :=-k\left(e_{d, \rho}^{n+1} \boldsymbol{f}\left(t_{n+1}\right), \overline{\boldsymbol{u}}_{h}\right)+\sum_{j=25}^{27}\left(\xi_{\boldsymbol{u}, j}^{n+1}, \overline{\boldsymbol{u}}_{h}\right) .
\end{aligned}
$$

Finally, from (11) and (19), we have

$$
\left(\nabla \cdot \boldsymbol{e}_{d, \boldsymbol{u}}^{n+1}, \bar{p}_{h}\right)=0 \quad \forall \bar{p}_{h} \in M_{h} .
$$

For simplicity of notation, let us denote the total consistency error as

$$
\left(\xi_{\boldsymbol{u}}^{n+1}, \overline{\boldsymbol{u}}_{h}\right)=\sum_{j=1}^{27}\left(\xi_{\boldsymbol{u}, j}^{n+1}, \overline{\boldsymbol{u}}_{h}\right) \quad \text { and } \quad\left(\zeta_{\boldsymbol{u}}^{n+1}, \overline{\boldsymbol{u}}_{h}\right)=\sum_{j=1}^{4}\left(\zeta_{\boldsymbol{u}, j}^{n+1}, \overline{\boldsymbol{u}}_{h}\right) .
$$

Therefore, we get the following variational formulation for $\left(\boldsymbol{e}_{d, \boldsymbol{u}}^{n+1}, e_{d, p}^{n+1}\right)$ :

$$
\left\{\begin{array}{c}
k\left(\rho_{h}^{n} \delta_{t} \boldsymbol{e}_{d, \boldsymbol{u}}^{n+1}, \overline{\boldsymbol{u}}_{h}\right)+k\left(\left(\left(\rho_{h}^{n+1} \boldsymbol{u}_{h}^{n}-\lambda \nabla \rho_{h}^{n+1}\right) \cdot \nabla\right) \boldsymbol{e}_{d, \boldsymbol{u}}^{n+1}, \overline{\boldsymbol{u}}_{h}\right)+k a\left(\rho_{h}^{n+1}, \boldsymbol{e}_{d, \boldsymbol{u}}^{n+1}, \overline{\boldsymbol{u}}_{h}\right) \\
+\frac{k}{2}\left(\nabla \cdot \boldsymbol{u}_{h}^{n} \rho_{h}^{n+1}, \boldsymbol{e}_{d, \boldsymbol{u}}^{n+1} \cdot \overline{\boldsymbol{u}}_{h}\right)-k\left(e_{d, p}^{n+1}, \nabla \cdot \overline{\boldsymbol{u}}_{h}\right)+\left(\zeta_{\boldsymbol{u}}^{n+1}, \overline{\boldsymbol{u}}_{h}\right)+\left(\xi_{\boldsymbol{u}}^{n+1}, \overline{\boldsymbol{u}}_{h}\right)=0, \\
\left(\nabla \cdot \boldsymbol{e}_{d, \boldsymbol{u}}^{n+1}, \bar{p}_{h}\right)=0,
\end{array}\right.
$$

for all $\left(\overline{\boldsymbol{u}}_{h}, p_{h}\right) \in \boldsymbol{V}_{h} \times M_{h}$.

Note that we have arrived at an error equation for the velocity that does not involve second derivative in time for the velocity as was announced before.

\subsection{Error equation for the density}

One easily sees that the above decomposition argument can be applied in the context of the density equation. Let us define the consistency errors associated to the density equation.

$$
\begin{aligned}
& k \delta_{t}\left(\rho_{h}^{n+1}-\rho\left(t_{n+1}\right), \bar{\rho}_{h}\right)=k\left(\delta_{t} \boldsymbol{e}_{d, \rho}^{n+1}, \bar{\rho}_{h}\right)+k\left(\delta_{t} \boldsymbol{e}_{i, \rho}^{n+1}, \bar{\rho}_{h}\right) \\
& :=k\left(\delta_{t} \boldsymbol{e}_{d, \rho}^{n+1}, \overline{\boldsymbol{u}}_{h}\right)+\left(\xi_{\rho, 1}^{n+1}, \bar{\rho}_{h}\right), \\
& \int_{t_{n}}^{t_{n+1}}\left(\boldsymbol{u}_{h}^{n} \cdot \nabla \rho_{h}^{n+1}-\boldsymbol{u}(s) \cdot \nabla \rho(s), \bar{\rho}_{h}\right) d s \\
& \quad=k\left(\boldsymbol{u}_{h}^{n} \cdot \nabla e_{d, \rho}^{n+1}, \bar{\rho}_{h}\right)+k\left(\boldsymbol{e}_{d, \boldsymbol{u}}^{n} \cdot \nabla K_{h} \rho\left(t_{n+1}\right), \bar{\rho}_{h}\right)
\end{aligned}
$$




$$
\begin{aligned}
& +\int_{t_{n}}^{t_{n+1}}\left(I_{h} \boldsymbol{u}\left(t_{n}\right) \cdot \nabla\left[K_{h} \rho\left(t_{n+1}\right)-K_{h} \rho(s)\right], \bar{\rho}_{h}\right) d s \\
& +\int_{t_{n}}^{t_{n+1}}\left(\left(I_{h} \boldsymbol{u}\left(t_{n}\right) \cdot \nabla\left[K_{h} \rho(s)-\rho(s)\right], \bar{\rho}_{h}\right) d s\right. \\
& +\int_{t_{n}}^{t_{n+1}}\left(\left[I_{h} \boldsymbol{u}\left(t_{n}\right)-I_{h} \boldsymbol{u}(s)\right] \cdot \nabla \rho(s), \bar{\rho}_{h}\right) d s \\
& +\int_{t_{n}}^{t_{n+1}}\left(\left[I_{h} \boldsymbol{u}(s)-\boldsymbol{u}(s)\right] \cdot \nabla \rho(s), \bar{\rho}_{h}\right) d s \\
& :=\sum_{j=1}^{2}\left(\zeta_{\rho, j}^{n+1}, \bar{\rho}_{h}\right)+\sum_{j=2}^{5}\left(\xi_{\rho, j}^{n+1}, \bar{\rho}_{h}\right), \\
& \lambda \int_{t_{n}}^{t_{n+1}}\left(\nabla \rho_{h}^{n+1}-\nabla \rho(s), \nabla \bar{\rho}_{h}\right) d s=\lambda k\left(\nabla e_{d, \rho}^{n+1}, \nabla \bar{\rho}_{h}\right) \\
& +\lambda \int_{t_{n}}^{t_{n+1}}\left(\nabla K_{h} \rho\left(t_{n+1}\right)-\nabla K_{h} \rho(s), \nabla \bar{\rho}_{h}\right) d s \\
& +\lambda \int_{t_{n}}^{t_{n+1}}\left(\nabla K_{h} \rho(s)-\nabla \rho(s), \nabla \bar{\rho}_{h}\right) d s \\
& :=\lambda k\left(\nabla e_{d, \rho}^{n+1}, \nabla \bar{\rho}_{h}\right)+\sum_{j=6}^{7}\left(\xi_{\rho, j}^{n+1}, \bar{\rho}_{h}\right) \text {. }
\end{aligned}
$$

Therefore, we get the following variational formulation for the density discrete error $e_{d, \rho}^{n+1}$ :

$$
k\left(\delta_{t} e_{d, \rho}^{n+1}, \bar{\rho}_{h}\right)+\lambda k\left(\nabla e_{d, \rho}^{n+1}, \nabla \bar{\rho}_{h}\right)+\left(\zeta_{\rho}^{n+1}, \bar{\rho}_{h}\right)+\left(\xi_{\rho}^{n+1}, \bar{\rho}_{h}\right)=0
$$

where

$$
\left(\zeta_{\rho}^{n+1}, \bar{\rho}_{h}\right)=\sum_{i=1}^{2}\left(\zeta_{\rho, i}^{n+1}, \bar{\rho}_{h}\right) \quad \text { and } \quad\left(\xi_{\rho}^{n+1}, \bar{\rho}_{h}\right)=\sum_{i=1}^{7}\left(\xi_{\rho, i}^{n+1}, \bar{\rho}_{h}\right)
$$




\subsection{Error estimates in energy norms}

The following lemma provides some estimates at each time step which will be fundamental to obtain the rate of convergence by an induction process.

Lemma 8 Suppose that $0<\widetilde{m} \leq \rho_{h}^{n}, \rho_{h}^{n+1} \leq \widetilde{M}$ in $\Omega$. Then, for $k$ small enough, there exists a constant $A>0$, independent of $(h, k)$ and $n$, such that the following inequality holds:

$$
\left\{\begin{array}{l}
\left(\left|\sqrt{\rho_{h}^{n+1}} \boldsymbol{e}_{d, \boldsymbol{u}}^{n+1}\right|^{2}+A\left|e_{d, \rho}^{n+1}\right|^{2}\right)-\left(\left|\sqrt{\rho_{h}^{n}} \boldsymbol{e}_{d, \boldsymbol{u}}^{n}\right|^{2}+A\left|e_{d, \rho}^{n}\right|^{2}\right) \\
+\left(\frac{\tilde{m}}{2}\left|\boldsymbol{e}_{d, \boldsymbol{u}}^{n+1}-\boldsymbol{e}_{d, \boldsymbol{u}}^{n}\right|^{2}+\frac{A}{2}\left|e_{d, \rho}^{n+1}-e_{d, \rho}^{n}\right|^{2}\right)+k \frac{3}{4}\left(\mu_{1}\left|\nabla \boldsymbol{e}_{d, \boldsymbol{u}}^{n+1}\right|^{2}+A \lambda\left|\nabla e_{d, \rho}^{n+1}\right|^{2}\right) \\
\leq G_{1} k\left(\tilde{m}\left|\boldsymbol{e}_{d, \boldsymbol{u}}^{n}\right|^{2}+A\left|e_{d, \rho}^{n}\right|^{2}\right)+k \frac{1}{4}\left(\mu_{1}\left|\nabla \boldsymbol{e}_{d, \boldsymbol{u}}^{n}\right|^{2}+A \lambda\left|\nabla e_{d, \rho}^{n}\right|^{2}\right) \\
+C\left(h^{2}+k^{2}\right)\left(\left\|\rho_{t}\right\|_{L^{2}\left(I_{n+1} ; H^{1}(\Omega)\right)}^{2}+\left\|\boldsymbol{u}_{t}\right\|_{L^{2}\left(I_{n+1} ; H^{1}(\Omega)\right)}^{2}\right) \\
+C h^{2}\left(\|\boldsymbol{u}\|_{L^{2}\left(I_{n+1} ; H^{2}(\Omega)\right)}^{2}+\|\rho\|_{L^{2}\left(I_{n+1} ; H^{2}(\Omega)\right)}^{2}\right),
\end{array}\right.
$$

where $\mu_{1}$ is the coercivity constant defined in (13), and $C, G_{1}$ are positive constants independent of $(h, k)$ and $n$.

Proof Stability and approximation properties of the interpolator operators $I_{h}, J_{h}$ and $K_{h}$ given in Lemma 4 must be kept in mind along the proof, since we will make use of them repeatedly.

First, let us take $\bar{\rho}_{h}=2 e_{d, \rho}^{n+1}$ as a test function into (32). Then, by using the identity $(a-b, 2 a)=a^{2}-b^{2}+(a-b)^{2}$, we obtain

$$
\begin{aligned}
& \left|e_{d, \rho}^{n+1}\right|^{2}-\left|e_{d, \rho}^{n}\right|^{2}+\left|e_{d, \rho}^{n+1}-e_{d, \rho}^{n}\right|^{2}+2 \lambda k\left|\nabla e_{d, \rho}^{n+1}\right|^{2} \\
& \quad+2\left(\zeta_{\rho}^{n+1}, e_{d, \rho}^{n+1}\right)+2\left(\xi_{\rho}^{n+1}, e_{d, \rho}^{n+1}\right)=0 .
\end{aligned}
$$

Let us start by bounding $\left(\xi_{\rho}^{n+1}, e_{d, \rho}^{n+1}\right)$. For the sake of simplicity, some specific terms will only be bounded in detail. Note that $\left(\xi_{\rho, 1}^{n+1}, e_{d, \rho}^{n+1}\right)$ may be written as

$$
\begin{aligned}
\left(\xi_{\rho, 1}^{n+1}, e_{d, \rho}^{n+1}\right) & =\int_{t_{n}}^{t_{n+1}}\left(\rho_{t}(s)-K_{h} \rho_{t}(s), e_{d, \rho}^{n+1}\right) d s \\
& \leq C h k^{1 / 2}\left\|\rho_{t}\right\|_{L^{2}\left(I_{n+1} ; H^{1}(\Omega)\right)}\left|e_{d, \rho}^{n+1}\right|,
\end{aligned}
$$


where we have used the interpolation error of $K_{h}$ and Schwarz' inequality. For $\left(\xi_{\rho, 2}^{n+1}\right.$, $\left.e_{d, \rho}^{n+1}\right)$ Fubini's rule and the interpolation stability lead to

$$
\begin{aligned}
\left(\xi_{\rho, 2}^{n+1}, e_{d, \rho}^{n+1}\right) & =\left(I_{h} \boldsymbol{u}\left(t_{n}\right) \cdot \nabla K_{h} \int_{t_{n}}^{t_{n+1}}\left(\rho\left(t_{n+1}\right)-\rho(s)\right) d s, e_{d, \rho}^{n+1}\right) \\
& =\left(I_{h} \boldsymbol{u}\left(t_{n}\right) \cdot \nabla K_{h} \int_{t_{n}}^{t_{n+1}}\left(\int_{s}^{t_{n+1}} \rho_{t}(z) d z\right) d s, e_{d, \rho}^{n+1}\right) \\
& =\int_{t_{n}}^{t_{n+1}}\left(z-t_{n}\right)\left(I_{h} \boldsymbol{u}\left(t_{n}\right) \cdot \nabla K_{h} \rho_{t}(z), e_{d, \rho}^{n+1}\right) d z \\
& \leq \int_{t_{n}}^{t_{n+1}}\left(z-t_{n}\right)\left\|I_{h} \boldsymbol{u}\left(t_{n}\right)\right\|_{L^{\infty}(\Omega)}\left|\nabla K_{h} \rho_{t}(z)\right|\left|e_{d, \rho}^{n+1}\right| d z \\
& \leq C k^{3 / 2}\left\|\boldsymbol{u}\left(t_{n}\right)\right\|_{L^{\infty}(\Omega)}\left\|\rho_{t}\right\|_{L^{2}\left(I_{n+1} ; H^{1}(\Omega)\right)}\left|e_{d, \rho}^{n+1}\right| \\
& \leq C k^{3 / 2}\left\|\rho_{t}\right\|_{L^{2}\left(I_{n+1} ; H^{1}(\Omega)\right)}\left|e_{d, \rho}^{n+1}\right| .
\end{aligned}
$$

In estimating $\left(\xi_{\rho, 3}^{n+1}, e_{d, \rho}^{n+1}\right)$, we use the interpolation error in $H^{1}$-norm verified by $K_{h}$ to obtain

$$
\begin{aligned}
\left(\xi_{\rho, 3}^{n+1}, e_{d, \rho}^{n+1}\right) & \leq C \int_{t_{n}}^{t_{n+1}}\left\|I_{h} \boldsymbol{u}\left(t_{n}\right)\right\|_{L^{\infty}(\Omega)} h\|\rho(s)\|_{H^{2}(\Omega)}\left|e_{d, \rho}^{n+1}\right| d s \\
& \leq C h k^{1 / 2}\left\|\boldsymbol{u}\left(t_{n}\right)\right\|_{L^{\infty}(\Omega)}\|\rho\|_{L^{2}\left(I_{n+1} ; H^{2}(\Omega)\right)}\left|e_{d, \rho}^{n+1}\right| \\
& \leq C h k^{1 / 2}\|\rho\|_{L^{2}\left(I_{n+1} ; H^{2}(\Omega)\right)}\left|e_{d, \rho}^{n+1}\right| .
\end{aligned}
$$

As was done in (36) and (37), we find that

$$
\begin{aligned}
\left(\xi_{\rho, 4}^{n+1}, e_{d, \rho}^{n+1}\right) & \leq C k^{3 / 2}\left\|\boldsymbol{u}_{t}\right\|_{L^{2}\left(I_{n+1} ; H^{1}(\Omega)\right)}\|\rho\|_{L^{\infty}\left(I_{n+1} ; W^{1,3}(\Omega)\right)}\left|e_{d, \rho}^{n+1}\right| \\
& \leq C k^{3 / 2}\left\|\boldsymbol{u}_{t}\right\|_{L^{2}\left(I_{n+1} ; H^{1}(\Omega)\right)}\left|e_{d, \rho}^{n+1}\right|, \\
\left(\xi_{\rho, 6}^{n+1}, e_{d, \rho}^{n+1}\right) & \leq C k^{3 / 2}\left\|\rho_{t}\right\|_{L^{2}\left(I_{n+1} ; H^{1}(\Omega)\right)}\left|\nabla e_{d, \rho}^{n+1}\right| .
\end{aligned}
$$

and

$$
\begin{aligned}
& \left(\xi_{\rho, 5}^{n+1}, e_{d, \rho}^{n+1}\right) \leq C h k^{1 / 2}\|\boldsymbol{u}\|_{L^{2}\left(I_{n+1} ; H^{2}(\Omega)\right)}\left|e_{d, \rho}^{n+1}\right|, \\
& \left(\xi_{\rho, 7}^{n+1}, e_{d, \rho}^{n+1}\right) \leq C h k^{1 / 2}\|\rho\|_{L^{2}\left(I_{n+1} ; H^{2}(\Omega)\right)}\left|\nabla e_{d, \rho}^{n+1}\right|,
\end{aligned}
$$


We proceed now to estimate $\left(\zeta_{\rho}^{n+1}, \bar{\rho}_{h}\right)$. We first handle

$$
\left(\zeta_{\rho, 1}^{n+1}, e_{d, \rho}^{n+1}\right)=k\left(\boldsymbol{e}_{d, \boldsymbol{u}}^{n} \cdot \nabla e_{d, \rho}^{n+1}, e_{d, \rho}^{n+1}\right)+k\left(I_{h} \boldsymbol{u}\left(t_{n}\right) \cdot \nabla e_{d, \rho}^{n+1}, e_{d, \rho}^{n+1}\right)
$$

bounding the two terms as follows:

$$
\begin{aligned}
k\left(\boldsymbol{e}_{d, \boldsymbol{u}}^{n} \cdot \nabla e_{d, \rho}^{n+1}, e_{d, \rho}^{n+1}\right) & \leq C k\left|\boldsymbol{e}_{d, \boldsymbol{u}}^{n}\right|\left|\nabla e_{d, \rho}^{n+1}\right|\left\|e_{d, \rho}^{n+1}\right\|_{L^{\infty}(\Omega)} \leq C k\left|\boldsymbol{e}_{d, \boldsymbol{u}}^{n}\right|\left|\nabla e_{d, \rho}^{n+1}\right|, \\
k\left(I_{h} \boldsymbol{u}\left(t_{n}\right) \cdot \nabla e_{d, \rho}^{n+1}, e_{d, \rho}^{n+1}\right) & \leq C k\left\|I_{h} \boldsymbol{u}\left(t_{n}\right)\right\|_{L^{\infty}(\Omega)}\left|\nabla e_{d, \rho}^{n+1}\right|\left|e_{d, \rho}^{n+1}\right| \\
& \leq C k\left|\nabla e_{d, \rho}^{n+1}\right|\left|e_{d, \rho}^{n+1}\right|,
\end{aligned}
$$

Finally, we control

$$
\left(\zeta_{\rho, 2}^{n+1}, e_{d, \rho}^{n+1}\right) \leq 2 k\left\|\boldsymbol{e}_{d, \boldsymbol{u}}^{n}\right\|_{L^{6}(\Omega)}\left\|\nabla K_{h} \rho\left(t_{n+1}\right)\right\|_{L^{3}(\Omega)}\left|e_{d, \rho}^{n+1}\right| \leq C k\left|\nabla \boldsymbol{e}_{d, \boldsymbol{u}}^{n}\right|\left|e_{d, \rho}^{n+1}\right|
$$

Inserting the above estimates in (34) and using Young's inequality leads to

$$
\begin{aligned}
& \left|e_{d, \rho}^{n+1}\right|^{2}-\left|e_{d, \rho}^{n}\right|^{2}+\left|e_{d, \rho}^{n+1}-e_{d, \rho}^{n}\right|^{2}+\lambda k\left|\nabla e_{d, \rho}^{n+1}\right|^{2} \leq C k\left(\left|e_{d, \rho}^{n+1}\right|^{2}+\left|\boldsymbol{e}_{d, \boldsymbol{u}}^{n}\right|^{2}\right) \\
& \quad+\varepsilon \mu_{1} k\left|\nabla \boldsymbol{e}_{d, \boldsymbol{u}}^{n}\right|^{2}+C\left(h^{2}+k^{2}\right)\left\|\rho_{t}\right\|_{L^{2}\left(I_{n+1} ; H^{1}(\Omega)\right)}^{2}+C k^{2}\left\|\boldsymbol{u}_{t}\right\|_{L^{2}\left(I_{n+1} ; H^{1}(\Omega)\right)}^{2} \\
& \quad+C h^{2}\left(\|\boldsymbol{u}\|_{L^{2}\left(I_{n+1} ; H^{2}(\Omega)\right)}^{2}+\|\rho\|_{L^{2}\left(I_{n+1} ; H^{2}(\Omega)\right)}^{2}\right)
\end{aligned}
$$

where $\varepsilon>0$ is a constant to be chosen later, and $\mu_{1}$ is defined in (13).

We now turn to finding the error estimates for the discrete velocity. Take $\overline{\boldsymbol{u}}_{h}=\boldsymbol{e}_{d, \boldsymbol{u}}^{n+1}$ as a test function into (30), taking into account that $\left(e_{d, p}^{n+1}, \nabla \cdot \boldsymbol{e}_{d, \boldsymbol{u}}^{n+1}\right)=0$ (owing to (31)) and the coercivity of $a\left(\rho_{h}^{n+1}, \cdot, \cdot\right)$ given in (13) to obtain

$$
\left\{\begin{array}{l}
\left.k\left(\rho_{h}^{n} \delta_{t} \boldsymbol{e}_{d, \boldsymbol{u}}^{n+1}, \boldsymbol{e}_{d, \boldsymbol{u}}^{n+1}\right)+\frac{\mu_{1}}{2} k\left|\nabla \boldsymbol{e}_{d, \boldsymbol{u}}^{n+1}\right|^{2}+k\left(\left(\rho_{h}^{n+1} \boldsymbol{u}_{h}^{n}-\lambda \nabla \rho_{h}^{n+1}\right) \cdot \nabla\right) \boldsymbol{e}_{d, \boldsymbol{u}}^{n+1}, \boldsymbol{e}_{d, \boldsymbol{u}}^{n+1}\right) \\
+\frac{1}{2} k\left(\nabla \cdot \boldsymbol{u}_{h}^{n} \rho_{h}^{n+1}, \boldsymbol{e}_{d, \boldsymbol{u}}^{n+1} \cdot \boldsymbol{e}_{d, \boldsymbol{u}}^{n+1}\right)+\left(\zeta_{\boldsymbol{u}}^{n+1}, \boldsymbol{e}_{d, \boldsymbol{u}}^{n+1}\right)+\left(\xi_{\boldsymbol{u}}^{n+1}, \boldsymbol{e}_{d, \boldsymbol{u}}^{n+1}\right) \leq 0 .
\end{array}\right.
$$

Let us handle (43) a little more. We pick $\bar{\rho}_{h}=\frac{k}{2}\left|\boldsymbol{e}_{d, \boldsymbol{u}}^{n+1}\right|^{2}$ to be a test function in (9) (which is possible owing to the hypothesis $\boldsymbol{V}_{h} \cdot \boldsymbol{V}_{h} \subseteq W_{h}$ imposed in (H4)). Then integration by parts yields

$$
\begin{aligned}
& \frac{k}{2}\left(\delta_{t} \rho_{h}^{n+1},\left|\boldsymbol{e}_{d, \boldsymbol{u}}^{n+1}\right|^{2}\right)-\frac{k}{2}\left(\rho_{h}^{n+1} \boldsymbol{u}_{h}^{n}-\lambda \nabla \rho_{h}^{n+1}, \nabla\left|\boldsymbol{e}_{d, \boldsymbol{u}}^{n+1}\right|^{2}\right) \\
& \quad-\frac{k}{2}\left(\nabla \cdot \boldsymbol{u}_{h}^{n} \rho_{h}^{n+1},\left|\boldsymbol{e}_{d, \boldsymbol{u}}^{n+1}\right|^{2}\right)=0 .
\end{aligned}
$$


If we now sum (44) to (43) combined with the discrete version of the time derivative given in Remark 1, we arrive at

$$
\begin{aligned}
& \left|\sqrt{\rho_{h}^{n+1}} \boldsymbol{e}_{d, \boldsymbol{u}}^{n+1}\right|^{2}-\left|\sqrt{\rho_{h}^{n}} \boldsymbol{e}_{d, \boldsymbol{u}}^{n}\right|^{2}+\left|\sqrt{\rho_{h}^{n}}\left(\boldsymbol{e}_{d, \boldsymbol{u}}^{n+1}-\boldsymbol{e}_{d, \boldsymbol{u}}^{n}\right)\right|^{2}+\mu_{1} k\left|\nabla \boldsymbol{e}_{d, \boldsymbol{u}}^{n+1}\right|^{2} \\
& \quad+2\left(\zeta_{\boldsymbol{u}}^{n+1}, \boldsymbol{e}_{d, \boldsymbol{u}}^{n+1}\right)+2\left(\xi_{\boldsymbol{u}}^{n+1}, \boldsymbol{e}_{d, \boldsymbol{u}}^{n+1}\right) \leq 0
\end{aligned}
$$

Next, we must bound adequately $\left(\zeta_{\boldsymbol{u}}^{n+1}, \boldsymbol{e}_{d, \boldsymbol{u}}^{n+1}\right)$ and $\left(\xi_{\boldsymbol{u}}^{n+1}, \boldsymbol{e}_{d, \boldsymbol{u}}^{n+1}\right)$. Let us take up the estimates of $\left(\xi_{\boldsymbol{u}}^{n+1}, \boldsymbol{e}_{d, \boldsymbol{u}}^{n+1}\right)$. Fubini's rule, and Hölder's and Sobolev's inequality show that

$$
\begin{aligned}
\left(\xi_{\boldsymbol{u}, 1}^{n+1}, \boldsymbol{e}_{d, \boldsymbol{u}}^{n+1}\right) & =\int_{t_{n}}^{t^{n+1}} \int_{t_{n}}^{s}\left(\rho_{t}(z) \boldsymbol{u}_{t}(s), \boldsymbol{e}_{d, \boldsymbol{u}}^{n+1}\right) d z d s \\
& \leq \int_{t_{n}}^{t^{n+1}} \int_{t_{n}}^{s}\left\|\rho_{t}(z)\right\|_{L^{3}(\Omega)}\left|\boldsymbol{u}_{t}(s)\right|\left\|\boldsymbol{e}_{d, \boldsymbol{u}}^{n+1}\right\|_{L^{6}(\Omega)} d z d s \\
& \leq C\left\|\boldsymbol{u}_{t}\right\|_{L^{\infty}\left(I_{n+1} ; L^{2}(\Omega)\right)}\left(\int_{t_{n}}^{t^{n+1}}\left(t_{n+1}-z\right)\left\|\rho_{t}(z)\right\|_{L^{3}(\Omega)} d z\right)\left|\nabla \boldsymbol{e}_{d, \boldsymbol{u}}^{n+1}\right| \\
& \leq C k^{3 / 2}\left\|\rho_{t}\right\|_{L^{2}\left(I_{n} ; L^{3}(\Omega)\right)}\left|\nabla \boldsymbol{e}_{d, \boldsymbol{u}}^{n+1}\right| .
\end{aligned}
$$

As in estimating (35), we have

$$
\left(\xi_{\boldsymbol{u}, 2}^{n+1}, \boldsymbol{e}_{d, \boldsymbol{u}}^{n+1}\right) \leq C h k^{1 / 2}\left\|\boldsymbol{u}_{t}\right\|_{L^{2}\left(I_{n+1} ; H^{1}(\Omega)\right)}\left|\boldsymbol{e}_{d, \boldsymbol{u}}^{n+1}\right|
$$

It is not hard to check from Hölder's and Sobolev's inequality that

$$
\begin{aligned}
\left(\xi_{\boldsymbol{u}, 3}^{n+1}, \boldsymbol{e}_{d, \boldsymbol{u}}^{n+1}\right) & \leq \int_{t_{n}}^{t_{n+1}}\left\|e_{d, \rho}^{n}\right\|_{L^{3}(\Omega)}\left|\boldsymbol{u}_{t}(s)\right|\left\|\boldsymbol{e}_{d, \boldsymbol{u}}^{n+1}\right\|_{L^{6}(\Omega)} d s \\
& \leq C k\left(\left|e_{d, \rho}^{n}\right|\left|\nabla \boldsymbol{e}_{d, \boldsymbol{u}}^{n+1}\right|+\left|e_{d, \rho}^{n}\right|^{1 / 2}\left|\nabla e_{d, \rho}^{n+1}\right|^{1 / 2}\left|\nabla \boldsymbol{e}_{d, \boldsymbol{u}}^{n+1}\right|\right) \\
\left(\xi_{\boldsymbol{u}, 4}^{n+1}, \boldsymbol{e}_{d, \boldsymbol{u}}^{n+1}\right) & \leq \int_{t_{n}}^{t_{n+1}}\left\|e_{i, \rho}^{n}\right\|_{L^{3}(\Omega)}\left|\boldsymbol{u}_{t}(s)\right|\left\|\boldsymbol{e}_{d, \boldsymbol{u}}^{n+1}\right\|_{L^{6}(\Omega)} d s \\
& \leq C h^{3 / 2} k^{1 / 2}\|\rho\|_{L^{2}\left(I_{n} ; H^{2}(\Omega)\right)}\left|\nabla \boldsymbol{e}_{d, \boldsymbol{u}}^{n+1}\right|
\end{aligned}
$$


To bound $\left(\xi_{\boldsymbol{u}, 5}^{n+1}, \boldsymbol{e}_{d, \boldsymbol{u}}^{n+1}\right)$ and $\left(\xi_{\boldsymbol{u}, 6}^{n+1}, \boldsymbol{e}_{d, \boldsymbol{u}}^{n+1}\right)$, we use the fact that $\boldsymbol{u} \in L^{\infty}\left(0, T ; \boldsymbol{W}_{0}^{1,3}(\Omega) \cap\right.$ $\boldsymbol{L}^{\infty}(\Omega)$ ), and mimic (36) to get

$$
\begin{aligned}
& \left(\xi_{\boldsymbol{u}, 5}^{n+1}, \boldsymbol{e}_{d, \boldsymbol{u}}^{n+1}\right) \leq C k^{2}\left|\nabla \boldsymbol{e}_{d, \boldsymbol{u}}^{n+1}\right|, \\
& \left(\xi_{\boldsymbol{u}, 6}^{n+1}, \boldsymbol{e}_{d, \boldsymbol{u}}^{n+1}\right) \leq C k^{3 / 2}\left\|\rho_{t}\right\|_{L^{2}\left(I_{n+1} ; L^{2}(\Omega)\right)}\left|\nabla \boldsymbol{e}_{d, \boldsymbol{u}}^{n+1}\right|, \\
& \left(\xi_{\boldsymbol{u}, 10}^{n+1}, \boldsymbol{e}_{d, \boldsymbol{u}}^{n+1}\right) \leq C h k^{1 / 2}\|\boldsymbol{u}\|_{L^{2}\left(I_{n+1} ; H^{2}(\Omega)\right)}\left|\nabla e_{d, \boldsymbol{u}}^{n+1}\right|
\end{aligned}
$$

In the same way as (37) we bound

$$
\begin{aligned}
& \left(\xi_{\boldsymbol{u}, 7}^{n+1}, \boldsymbol{e}_{d, \boldsymbol{u}}^{n+1}\right) \leq C h^{2} k^{1 / 2}\|\boldsymbol{u}\|_{L^{2}\left(I_{n+1} ; H^{2}(\Omega)\right)}\left|\nabla \boldsymbol{e}_{d, \boldsymbol{u}}^{n+1}\right|, \\
& \left(\xi_{\boldsymbol{u}, 8}^{n+1}, \boldsymbol{e}_{d, \boldsymbol{u}}^{n+1}\right) \leq C h^{2} k^{1 / 2}\|\rho\|_{L^{2}\left(I_{n+1} ; H^{2}(\Omega)\right)}\left|\nabla \boldsymbol{e}_{d, \boldsymbol{u}}^{n+1}\right| .
\end{aligned}
$$

Analogously to (39), it follows that

$$
\left(\xi_{\boldsymbol{u}, 9}^{n+1}, \boldsymbol{e}_{d, \boldsymbol{u}}^{n+1}\right) \leq C k^{3 / 2}\left\|\boldsymbol{u}_{t}\right\|_{L^{2}\left(I_{n+1} ; H^{1}(\Omega)\right)}\left|e_{d, \boldsymbol{u}}^{n+1}\right|
$$

An argument similar to the convective term for the velocity also shows

$$
\begin{aligned}
& \left(\xi_{\boldsymbol{u}, 11}^{n+1}, \boldsymbol{e}_{d, \boldsymbol{u}}^{n+1}\right) \leq C k^{3 / 2}\left\|\rho_{t}\right\|_{L^{2}\left(I_{n+1} ; H^{1}(\Omega)\right)}\left|\nabla e_{d, \boldsymbol{u}}^{n+1}\right|, \\
& \left(\xi_{\boldsymbol{u}, 12}^{n+1}, \boldsymbol{e}_{d, \boldsymbol{u}}^{n+1}\right) \leq C h k^{1 / 2}\|\rho\|_{L^{2}\left(I_{n+1} ; H^{2}(\Omega)\right)}\left|\nabla e_{d, \boldsymbol{u}}^{n+1}\right|, \\
& \left(\xi_{\boldsymbol{u}, 13}^{n+1}, \boldsymbol{e}_{d, \boldsymbol{u}}^{n+1}\right) \leq C k^{3 / 2}\left\|\boldsymbol{u}_{t}\right\|_{L^{2}\left(I_{n+1} ; H^{1}(\Omega)\right)}\left|\nabla e_{d, \boldsymbol{u}}^{n+1}\right|, \\
& \left(\xi_{\boldsymbol{u}, 14}^{n+1}, \boldsymbol{e}_{d, \boldsymbol{u}}^{n+1}\right) \leq C h k^{1 / 2}\|\boldsymbol{u}\|_{L^{2}\left(I_{n+1} ; H^{2}(\Omega)\right)}\left|\nabla e_{d, \boldsymbol{u}}^{n+1}\right|,
\end{aligned}
$$

where we have used the fact that $\rho \in L^{\infty}\left(0, T ; W^{1,3}(\Omega)\right)$ in the last two lines. One sees readily that

$$
\left(\xi_{\boldsymbol{u}, 15}^{n+1}, \boldsymbol{e}_{d, \boldsymbol{u}}^{n+1}\right) \leq C k^{3 / 2}\left\|\boldsymbol{u}_{t}\right\|_{L^{2}\left(I_{n+1} ; H^{1}(\Omega)\right)}\left|\nabla e_{d, \boldsymbol{u}}^{n+1}\right|,
$$

and

$$
\left(\xi_{\boldsymbol{u}, 16}^{n+1}, \boldsymbol{e}_{d, \boldsymbol{u}}^{n+1}\right) \leq C h k^{1 / 2}\|\boldsymbol{u}\|_{L^{2}\left(I_{n+1} ; H^{2}(\Omega)\right)}\left|\nabla e_{d, \boldsymbol{u}}^{n+1}\right|
$$

The other $\lambda$-terms may be bounded as

$$
\begin{aligned}
& \left(\xi_{\boldsymbol{u}, 17}^{n+1}, \boldsymbol{e}_{d, \boldsymbol{u}}^{n+1}\right) \leq C k^{3 / 2}\left\|\rho_{t}\right\|_{L^{2}\left(I_{n+1} ; H^{1}(\Omega)\right)}\left|\nabla e_{d, \boldsymbol{u}}^{n+1}\right|, \\
& \left(\xi_{\boldsymbol{u}, 18}^{n+1}, \boldsymbol{e}_{d, \boldsymbol{u}}^{n+1}\right) \leq C h k^{1 / 2}\|\rho\|_{L^{2}\left(I_{n+1} ; H^{2}(\Omega)\right)}\left|\nabla e_{d, \boldsymbol{u}}^{n+1}\right|,
\end{aligned}
$$




$$
\begin{aligned}
\left(\xi_{\boldsymbol{u}, 19}^{n+1}, \boldsymbol{e}_{d, \boldsymbol{u}}^{n+1}\right) & \leq C k^{3 / 2}\left\|\boldsymbol{u}_{t}\right\|_{L^{2}\left(I_{n+1} ; H^{1}(\Omega)\right)}\left|\nabla e_{d, \boldsymbol{u}}^{n+1}\right|, \\
\left(\xi_{\boldsymbol{u}, 20}^{n+1}, \boldsymbol{e}_{d, \boldsymbol{u}}^{n+1}\right) & \leq C h k^{1 / 2}\|\boldsymbol{u}\|_{L^{2}\left(I_{n+1} ; H^{2}(\Omega)\right)}\left|\nabla e_{d, \boldsymbol{u}}^{n+1}\right|,
\end{aligned}
$$

and the terms coming from the stabilizing term remain bounded as

$$
\begin{aligned}
\left(\xi_{\boldsymbol{u}, 21}^{n+1}, \boldsymbol{e}_{d, \boldsymbol{u}}^{n+1}\right) & \leq C h k^{1 / 2}\left\|\boldsymbol{u}_{t}\right\|_{L^{2}\left(I_{n+1} ; H^{1}(\Omega)\right)}\left|e_{d, \boldsymbol{u}}^{n+1}\right|, \\
\left(\xi_{\boldsymbol{u}, 22}^{n+1}, \boldsymbol{e}_{d, \boldsymbol{u}}^{n+1}\right) & \leq C h k^{1 / 2}\|\boldsymbol{u}\|_{L^{2}\left(I_{n+1} ; H^{2}(\Omega)\right)}\left|e_{d, \boldsymbol{u}}^{n+1}\right|
\end{aligned}
$$

Making use of the property (19) of $I_{h}$, we see that $\left(\xi_{\boldsymbol{u}, 23}^{n+1}, \boldsymbol{e}_{d, \boldsymbol{u}}^{n+1}\right)=0$. The other pressure term can be bounded as follows:

$$
\left(\xi_{\boldsymbol{u}, 24}^{n+1}, \boldsymbol{e}_{d, \boldsymbol{u}}^{n+1}\right) \leq C h k^{1 / 2}\|p\|_{L^{2}\left(I_{n+1} ; H^{1}(\Omega)\right)}\left|\nabla \cdot e_{d, \boldsymbol{u}}^{n+1}\right|,
$$

Finally, the forcing terms are estimated as

$$
\begin{aligned}
\left(\xi_{\boldsymbol{u}, 25}^{n+1}, \boldsymbol{e}_{d, \boldsymbol{u}}^{n+1}\right) & \leq C k^{3 / 2}\left\|\rho_{t}\right\|_{L^{2}\left(I_{n+1} ; L^{3}(\Omega)\right)}\left\|\boldsymbol{f}\left(t_{n+1}\right)\right\|_{L^{2}(\Omega)}\left|\nabla e_{d, \boldsymbol{u}}^{n+1}\right| \\
& \leq C k^{3 / 2}\left\|\rho_{t}\right\|_{L^{2}\left(I_{n+1} ; L^{3}(\Omega)\right)}\left|\nabla e_{d, \boldsymbol{u}}^{n+1}\right|, \\
\left(\xi_{\boldsymbol{u}, 26}^{n+1}, \boldsymbol{e}_{d, \boldsymbol{u}}^{n+1}\right) & \leq C h k^{1 / 2}\|\boldsymbol{f}\|_{L^{2}\left(I_{n+1} ; L^{3}(\Omega)\right)}\left|\nabla e_{d, \boldsymbol{u}}^{n+1}\right|, \\
\left(\xi_{\boldsymbol{u}, 27}^{n+1}, \boldsymbol{e}_{d, \boldsymbol{u}}^{n+1}\right) & \leq C k^{3 / 2}\left\|\boldsymbol{f}_{t}\right\|_{L^{2}\left(I_{n+1} ; L^{6 / 5}(\Omega)\right)}\left|\nabla e_{d, \boldsymbol{u}}^{n+1}\right| .
\end{aligned}
$$

What remains to be bounded, to complete the estimates, is $\left(\zeta_{\boldsymbol{u}}^{n+1}, \boldsymbol{e}_{d, \boldsymbol{u}}^{n+1}\right)$ :

$$
\begin{aligned}
\left(\zeta_{\boldsymbol{u}, 1}^{n+1}, \boldsymbol{e}_{d, \boldsymbol{u}}^{n+1}\right) \leq & k \mid K_{h} \rho\left(t_{n+1}\right) \boldsymbol{e}_{d, \boldsymbol{u}}^{n}+e_{d, \rho}^{n+1} I_{h} \boldsymbol{u}\left(t_{n}\right) \\
& -\lambda \nabla e_{d, \rho}^{n+1} \mid\left\|\nabla I_{h} \boldsymbol{u}\left(t_{n+1}\right)\right\|_{L^{3}(\Omega)}\left\|\boldsymbol{e}_{d, \boldsymbol{u}}^{n+1}\right\|_{L^{6}(\Omega)} \\
\leq & C k\left(\left|\boldsymbol{e}_{d, \boldsymbol{u}}^{n}\right|^{2}+\left|e_{d, \rho}^{n+1}\right|^{2}+\left|\nabla e_{d, \rho}^{n+1}\right|^{2}\right)\left|\nabla \boldsymbol{e}_{d, \boldsymbol{u}}^{n+1}\right|^{2}, \\
\left(\zeta_{\boldsymbol{u}, 2}^{n+1}, \boldsymbol{e}_{d, \boldsymbol{u}}^{n+1}\right) \leq & k\left|\nabla e_{d, \rho}^{n+1}\right|\left\|\nabla I_{h} \boldsymbol{u}\left(t_{n+1}\right)\right\|_{L^{3}(\Omega)}\left\|\boldsymbol{e}_{d, \boldsymbol{u}}^{n+1}\right\|_{L^{6}(\Omega)} \\
\leq & C k\left|\nabla e_{d, \rho}^{n+1}\right|\left|\nabla \boldsymbol{e}_{d, \boldsymbol{u}}^{n+1}\right|, \\
\left(\zeta_{\boldsymbol{u}, 3}^{n+1}, \boldsymbol{e}_{d, \boldsymbol{u}}^{n+1}\right) \leq & C k\left\|e_{d, \rho}^{n+1}\right\|_{L^{6}(\Omega)}\left\|\nabla I_{h} \boldsymbol{u}\left(t_{n+1}\right)\right\|_{L^{3}(\Omega)}\left|\nabla \boldsymbol{e}_{d, \boldsymbol{u}}^{n+1}\right| \\
\leq & C k\left(\left|e_{d, \rho}^{n+1}\right|+\left|\nabla e_{d, \rho}^{n+1}\right|\right)\left|\nabla \boldsymbol{e}_{d, \boldsymbol{u}}^{n+1}\right|, \\
\left(\zeta_{\boldsymbol{u}, 4}^{n+1}, \boldsymbol{e}_{d, \boldsymbol{u}}^{n+1}\right) \leq & C k\left|\boldsymbol{e}_{d, \boldsymbol{u}}^{n+1}\right|\left|\nabla \boldsymbol{e}_{d, \boldsymbol{u}}^{n}\right| .
\end{aligned}
$$


Again, applying the above estimates in (43) and using Young's inequality yields

$$
\begin{aligned}
& \left|\sqrt{\rho_{h}^{n+1}} \boldsymbol{e}_{d, \boldsymbol{u}}^{n+1}\right|^{2}-\left|\sqrt{\rho_{h}^{n}} \boldsymbol{e}_{d, \boldsymbol{u}}^{n}\right|^{2}+\left|\sqrt{\rho_{h}^{n}}\left(\boldsymbol{e}_{d, \boldsymbol{u}}^{n+1}-\boldsymbol{e}_{d, \boldsymbol{u}}^{n}\right)\right|^{2}+\frac{\mu_{1} k}{2}\left|\nabla \boldsymbol{e}_{d, \boldsymbol{u}}^{n+1}\right|^{2} \\
& \leq C k\left(\left|e_{d, \rho}^{n+1}\right|^{2}+\left|e_{d, \rho}^{n}\right|^{2}+\left|\boldsymbol{e}_{d, \boldsymbol{u}}^{n+1}\right|^{2}+\left|\boldsymbol{e}_{d, \boldsymbol{u}}^{n}\right|^{2}+\left|\nabla e_{d, \rho}^{n+1}\right|\right)+\delta \lambda k\left|\nabla e_{d, \rho}^{n}\right|^{2} \\
& \quad+C k^{2}\left|\nabla \boldsymbol{e}_{d, \boldsymbol{u}}^{n+1}\right|+C k^{2}\left(\left\|\rho_{t}\right\|_{L^{2}\left(I_{n+1} ; H^{1}(\Omega)\right)}^{2}+\left\|\boldsymbol{u}_{t}\right\|_{L^{2}\left(I_{n+1} ; H^{1}(\Omega)\right)}^{2}\right) \\
& \quad+C h^{2}\left\|\boldsymbol{u}_{t}\right\|_{L^{2}\left(I_{n+1} ; H^{1}(\Omega)\right)}^{2}+C h^{3}\|\rho\|_{L^{2}\left(I_{n+1} ; H^{2}(\Omega)\right)}^{2} \\
& \quad+C h^{2}\left(\|\boldsymbol{u}\|_{L^{2}\left(I_{n+1} ; H^{2}(\Omega)\right)}^{2}+\|p\|_{L^{2}\left(I_{n+1} ; H^{1}(\Omega)\right)}^{2}+\|\rho\|_{L^{2}\left(I_{n+1} ; H^{2}(\Omega)\right)}^{2}\right) \\
& \quad+C h^{2}\|\boldsymbol{f}\|_{L^{2}\left(I_{n+1} ; L^{3}(\Omega)\right)}^{2}+C k^{2}\left\|\boldsymbol{f}_{t}\right\|_{L^{2}\left(I_{n+1} ; L^{6 / 5}(\Omega)\right)}^{2}
\end{aligned}
$$

where $\delta>0$ is a constant to be chosen later.

Finally, we bound $\left|e_{\rho}^{n+1}\right|^{2} \leq 2\left(\left|e_{\rho}^{n+1}-e_{\rho}^{n}\right|^{2}+\left|e_{\rho}^{n}\right|^{2}\right)$ and $\left|\boldsymbol{e}_{u}^{n+1}\right|^{2} \leq 2\left(\mid \boldsymbol{e}_{u}^{n+1}-\right.$ $\left.\boldsymbol{e}_{u}^{n}\right|^{2}+\left|\boldsymbol{e}_{u}^{n}\right|^{2}$ ) in (42) and (46) and balance inequalities (42) and (46) so that the term $C k\left|\nabla e_{\rho}^{n+1}\right|^{2}$ is absorbed on the right-hand side of (42). To end, take $k$ small enough so that the terms $C k\left|e_{d, \rho}^{n+1}-e_{d, \rho}^{n}\right|^{2}$ and $C k^{2}\left|\nabla \boldsymbol{e}_{d, \rho}^{n+1}\right|^{2}$, and $C k\left|\boldsymbol{e}_{d, \boldsymbol{u}}^{n+1}-\boldsymbol{e}_{d, \boldsymbol{u}}^{n}\right|^{2}$ and $C k^{2}\left|\nabla \boldsymbol{e}_{d, \boldsymbol{u}}^{n+1}\right|$ are controlled on the left-hand side of (42) and (46), respectively. Then the recursive inequality (33) holds automatically by choosing $\varepsilon$ and $\delta$ in the obvious way.

At this point, we can choose the approximation of the initial data. Our finiteelement method would start with, say, $\rho_{h}^{0}=K_{h} \rho_{0}$ and $\boldsymbol{u}_{h}^{0}=I_{h} \boldsymbol{u}_{0}$. With this choice we can say that

$$
\begin{aligned}
& \left|\boldsymbol{u}_{0}-\boldsymbol{u}_{h}^{0}\right| \leq C h, \quad\left|\nabla \boldsymbol{u}_{0}^{h}\right| \leq G_{2}, \\
& \left|\rho_{0}-\rho_{h}^{0}\right| \leq C h, \quad 0<\widetilde{m} \leq \rho_{h}^{0}(\boldsymbol{x}) \leq \widetilde{M} .
\end{aligned}
$$

Now, we are in position to prove existence of solution of the scheme and optimal error estimates in weak norms for discrete errors.

Theorem 9 Assume hypotheses $(H 0)-(H 4)$ constraint $(S)$ and $(h, k)$ small enough (in order to apply Lemmas 6, 5, Corollary 7 and Lemma 8). Then there exists a unique solution $\left(\rho_{h}^{n}, \boldsymbol{u}_{h}^{n}, p_{h}^{n}\right)$ of scheme (9)-(11) and the following estimates hold:

$$
\begin{aligned}
& 0<\tilde{m} \leq \rho_{h}^{n+1} \leq \tilde{M}, \quad \forall n: 0 \leq n \leq N-1, \\
& \left\{\begin{array}{l}
\max _{0 \leq n \leq N-1}\left(\widetilde{m}\left|\boldsymbol{e}_{d, \boldsymbol{u}}^{n+1}\right|^{2}+A\left|e_{d, \rho}^{n+1}\right|^{2}\right)+\sum_{n=0}^{N-1}\left(\frac{\tilde{m}}{2}\left|\boldsymbol{e}_{d, \boldsymbol{u}}^{n+1}-\boldsymbol{e}_{d, \boldsymbol{u}}^{n}\right|^{2}+\frac{A}{2}\left|e_{d, \rho}^{n+1}-e_{d, \rho}^{n}\right|^{2}\right) \\
+k \sum_{n=0}^{N-1}\left(\frac{\mu_{1}}{2}\left|\nabla \boldsymbol{e}_{d, \boldsymbol{u}}^{n+1}\right|^{2}+A \lambda\left|\nabla e_{d, \rho}^{n+1}\right|^{2}\right) \leq C\left(k^{2}+h^{2}\right) .
\end{array}\right.
\end{aligned}
$$


Proof If we assume that (26) and (33) hold for each $n=0, \ldots, N-1$, then (49) and (50) are readily satisfied. Indeed, (49) holds trivially. Next, observe that $e_{d, \rho}^{0}=0$ and $\boldsymbol{e}_{d, \boldsymbol{u}}^{0}=0$ by definition. Then, by summing up (33) for $n=0, \ldots, N-1$, applying the discrete Gronwall lemma, and taking into account (49), it follows (50) from the regularity for the exact solution given in $(H 0)$.

Let us therefore see that (26) and (33) hold by induction on $n$. For $n=0$ we have by hypothesis $0<\widetilde{m} \leq \rho_{h}^{0} \leq \widetilde{M}$, from (48), and $\left|\nabla \boldsymbol{u}_{h}^{0}\right| \leq G_{2}$, from (47). Let $C_{s}:=\max \left\{G_{3}, G_{2}\right\}$, where $G_{3}>0$ is a constant to be chosen later on.

By virtue of Corollary 7 , the point-wise estimate $0<\widetilde{m} \leq \rho_{h}^{1} \leq \widetilde{M}$ holds, that is, (26) is satisfied for $n=0$. Thus, from Lemma 8, we have (33) for $n=0$.

Suppose by induction that (26) and (33) holds for $l=0, \ldots, n-1$. Then, sum up (33) for $l=0, \ldots, n-1$ to get

$$
\begin{aligned}
& \widetilde{m}\left|\boldsymbol{e}_{d, \boldsymbol{u}}^{n}\right|^{2}+A\left|e_{d, \rho}^{n}\right|^{2}+\frac{k}{2} \sum_{l=1}^{n}\left(\mu_{1}\left|\nabla \boldsymbol{e}_{d, \boldsymbol{u}}^{l}\right|^{2}+A \lambda\left|\nabla \boldsymbol{e}_{d, \rho}^{l}\right|^{2}\right) \\
& \leq G_{1} k \sum_{l=0}^{n-1}\left(\widetilde{m}\left|\boldsymbol{e}_{d, \boldsymbol{u}}^{l}\right|^{2}+A\left|e_{d, \rho}^{l}\right|^{2}\right) \\
& +C\left(h^{2}+k^{2}\right) \sum_{l=0}^{n-1}\left(\left\|\rho_{t}\right\|_{L^{2}\left(I_{n+1} ; H^{1}(\Omega)\right)}^{2}+\left\|\boldsymbol{u}_{t}\right\|_{L^{2}\left(I_{n+1} ; H^{1}(\Omega)\right)}^{2}\right) \\
& +C h^{2} \sum_{l=0}^{n-1}\left(\|\boldsymbol{u}\|_{L^{2}\left(I_{n+1} ; H^{2}(\Omega)\right)}^{2}+\|\rho\|_{L^{2}\left(I_{n+1} ; H^{2}(\Omega)\right)}^{2}\right), \\
& \leq G_{1} k \sum_{l=0}^{n-1}\left(\widetilde{m}\left|\boldsymbol{e}_{d, \boldsymbol{u}}^{l}\right|^{2}+A\left|e_{d, \rho}^{l}\right|^{2}\right)+C\left(k^{2}+h^{2}\right),
\end{aligned}
$$

where $G_{1}$ is the constant appearing in Lemma 8 and $C>0$ is a constant depending on the exact solution. By applying the discrete Gronwall lemma, one obtains

$$
\frac{\mu_{1}}{2} k \sum_{l=1}^{n}\left|\nabla \boldsymbol{e}_{d, \boldsymbol{u}}^{l}\right|^{2} \leq C e^{G_{1} t_{n}}\left(k^{2}+h^{2}\right) \leq C e^{G_{1} T}\left(k^{2}+h^{2}\right) .
$$

Since $C e^{G_{1} T}$ is independent of $C_{s}$, it is easy to deduce that there exists a constant $G_{3}>0$ such that $\left|\nabla \boldsymbol{u}_{h}^{l}\right| \leq G_{3}$, for $l=1, \ldots, n$. Recall that $C_{s}:=\max \left\{G_{3}, G_{2}\right\}$, hence $\left|\nabla \boldsymbol{u}_{h}^{l}\right| \leq C_{s}$ for each $l=0, \ldots, n$. It is important to know that the constant $C_{s}$ is independent of $(h, k)$ and the time-steps $l$. Then, Corollary 7 implies (26) for $n$, that is $0<\widetilde{m} \leq \rho_{h}^{l+1} \leq \widetilde{M}$ for each $l=0, \ldots, n$. Finally, we can apply Lemma 8 and deduce (33) for $n$.

The final step to prove Theorem 2 is left to the reader since it only draws on the interpolation errors in Lemma 4. 
4.4 Error estimates for the density in strong norms

Before proceeding any further, we are going to define other interpolation operator for the density based on the Poisson-Neumann problem. For each $\rho \in H^{1}(\Omega)$, we set $K_{h} \rho \in W_{h}$ such that

$$
\left\{\begin{array}{l}
\left(\nabla\left(\rho-K_{h} \rho\right), \nabla \bar{\rho}_{h}\right)=0 \quad \forall \bar{\rho}_{h} \in W_{h}, \\
\int_{\Omega} K_{h} \rho=\int_{\Omega} \rho .
\end{array}\right.
$$

In fact, $K_{h} \rho$ can be obtained as follows:

1. Consider $\eta=\rho-\oint_{\Omega} \rho \in L_{0}^{2}(\Omega)$.

2. Find $\eta_{h} \in W_{h} \cap L_{0}^{2}(\Omega)$ as the solution of the discrete Poisson-Neumann problem:

$$
\left(\nabla\left(\eta-\eta_{h}\right), \nabla \bar{\rho}_{h}\right)=0, \quad \forall \bar{\rho}_{h} \in W_{h} \cap L_{0}^{2}(\Omega) .
$$

3. Calculate $K_{h} \rho=\eta_{h}+\oint_{\Omega} \rho$.

This interpolation operator $K_{h}$ holds the following error approximations (see [4,7])

$$
\left\|\rho-K_{h} \rho\right\|_{H^{1}(\Omega)} \leq C h\|\rho\|_{H^{2}(\Omega)} \quad \forall \rho \in H^{2}(\Omega) .
$$

Consider the discrete Laplacian operator $\Delta_{h}: W_{h} \rightarrow W_{h}$ defined as the solution to the problem

$$
\Delta_{h} \rho_{h} \in W_{h} \quad \text { such that } \quad\left(-\Delta_{h} \rho_{h}, \bar{\rho}_{h}\right)=\left(\nabla \rho_{h}, \nabla \bar{\rho}_{h}\right) \quad \forall \bar{\rho}_{h} \in W_{h} .
$$

Thus, we see that the discrete density equation (9) can be written in terms of this operator as:

$$
\left(\frac{\rho_{h}^{n+1}-\rho_{h}^{n}}{k}, \bar{\rho}_{h}\right)+\left(\boldsymbol{u}_{h}^{n} \cdot \nabla \rho_{h}^{n+1}, \bar{\rho}_{h}\right)-\lambda\left(\Delta_{h} \rho_{h}^{n+1}, \bar{\rho}_{h}\right)=0 \quad \forall \bar{\rho}_{h} \in W_{h}
$$

Let $P_{h}$ be the $L^{2}$-orthogonal projection from $L^{2}(\Omega)$ onto $W_{h}$, and consider the error for $\Delta \rho$ :

$$
e_{\Delta}^{n+1}=\Delta \rho\left(t_{n+1}\right)-\Delta_{h} \rho_{h}^{n+1},
$$

which we again decompose as $e_{\Delta}^{n+1}=e_{i, \Delta}^{n+1}+e_{d, \Delta}^{n+1}$ with

$$
e_{d, \Delta}^{n+1}=P_{h}\left(\Delta \rho\left(t_{n+1}\right)\right)-\Delta_{h} \rho_{h}^{n+1} \text { and } \quad e_{i, \Delta}^{n+1}=\Delta \rho\left(t_{n+1}\right)-P_{h}\left(\Delta \rho\left(t_{n+1}\right)\right) .
$$


On the other hand, although the interpolation operator related to density has changed, for simplicity, the corresponding error will be denoted in the same manner $e_{\rho}^{n}=$ $e_{i, \rho}^{n}+e_{d, \rho}^{n}$. Then the error equation for the density can be stated as

$$
k\left(\delta_{t} e_{d, \rho}^{n+1}, \bar{\rho}_{h}\right)+\lambda k\left(e_{d, \Delta}^{n+1}, \bar{\rho}_{h}\right)+\left(\zeta_{\rho}^{n+1}, \bar{\rho}_{h}\right)+\left(\xi_{\rho}^{n+1}, \bar{\rho}_{h}\right)=0,
$$

where now the diffusion consistency error takes the form

$$
\begin{aligned}
\sum_{i=6}^{7}\left(\xi_{\rho, i}^{n+1}, \bar{\rho}_{h}\right)= & \lambda \int_{t_{n}}^{t_{n+1}}\left(P_{h}\left(-\Delta \rho\left(t_{n+1}\right)\right)-P_{h}(-\Delta \rho(s)), \bar{\rho}_{h}\right) d s \\
& +\lambda \int_{t_{n}}^{t_{n+1}}\left(P_{h}(-\Delta \rho(s))+\Delta \rho(s), \bar{\rho}_{h}\right) d s .
\end{aligned}
$$

Note that $\left(\xi_{\rho, 7}^{n+1}, \bar{\rho}_{h}\right)=0$ by definition of $P_{h}$.

For fixed $h$, let $e^{n+1}(h) \in H^{2}(\Omega)$ be the solution of the auxiliary problem

$$
-\Delta e^{n+1}(h)=e_{d, \Delta}^{n+1} \text { in } \Omega,\left.\quad \frac{\partial e^{n+1}(h)}{\partial \boldsymbol{n}}\right|_{\partial \Omega}=0, \quad \int_{\Omega} e^{n+1}(h)=0 .
$$

Since the $H^{2}$-regularity of (56) (see hypothesis $(H 1)$ ) and $\int_{\Omega} e_{d, \Delta}^{n+1}=0$ hold, it is guaranteed that (56) is well-posed. The function $e^{n+1}(h)$ can be seen as a continuous approximation of $e_{d, \Delta}^{n+1}$ as the following result shows.

Lemma 10 It follows that

$$
\left|\nabla\left(e_{d, \rho}^{n+1}-e^{n+1}(h)\right)\right| \leq C h\left|e_{d, \Delta}^{n+1}\right| .
$$

where $C>0$ is a constant independent of $(k, h)$, and of the step $n$.

Proof We first state that $e_{d, \rho}^{n+1} \in W_{h}$ satisfies the equation

$$
\left(\nabla e_{d, \rho}^{n+1}, \nabla \bar{\rho}_{h}\right)=\left(e_{d, \Delta}^{n+1}, \bar{\rho}_{h}\right) \quad \forall \bar{\rho}_{h} \in W_{h} .
$$

Indeed, on one hand, in view of definition of $-\Delta_{h}$ given in (54), we find that

$$
\left(\nabla \rho_{h}^{n+1}, \nabla \bar{\rho}_{h}\right)=-\left(\Delta_{h} \rho_{h}^{n+1}, \bar{\rho}_{h}\right) \quad \forall \bar{\rho}_{h} \in W_{h}
$$

On the other hand, from the definition of the interpolation operators $K_{h}$ and $P_{h}$, one easily sees that

$\left(\nabla K_{h} \rho\left(t_{n+1}\right), \nabla \bar{\rho}_{h}\right)=\left(\nabla \rho\left(t_{n+1}\right), \nabla \bar{\rho}_{h}\right)=\left(-\Delta \rho\left(t_{n+1}\right), \bar{\rho}_{h}\right)=\left(P_{h}\left(-\Delta \rho\left(t_{n+1}\right)\right), \bar{\rho}_{h}\right)$ 
holds since $\rho\left(t_{n+1}\right) \in H_{N}^{2}(\Omega)$. By subtracting both equalities, one finds (58). In particular, by comparing (56) and (58)

$$
\left(\nabla\left(e_{d, \rho}^{n+1}-e^{n+1}(h)\right), \nabla \bar{\rho}_{h}\right)=\forall \bar{\rho}_{h} \in W_{h} .
$$

Finally, to obtain (57) we take $\bar{\rho}_{h}=e_{d, \rho}^{n+1}-e^{n+1}(h)+e^{n+1}(h)-K_{h} e^{n+1}(h)$ as a test function and estimate by

$$
\begin{aligned}
\left|\nabla\left(e_{d, \rho}^{n+1}-e^{n+1}(h)\right)\right| & \leq\left|\nabla\left(e^{n+1}(h)-K_{h} e^{n+1}(h)\right)\right| \leq C h\left\|e^{n+1}(h)\right\|_{H^{2}(\Omega)} \\
& \leq C h\left|e_{d, \Delta}^{n+1}\right|,
\end{aligned}
$$

where we have used the approximation property (53) and the $H^{2}$-continuous dependency of problem (56), $\left\|e^{n+1}(h)\right\|_{H^{2}(\Omega)} \leq C\left|e_{d, \Delta}^{n+1}\right|$ (imposed in (H1)).

Theorem 11 Under conditions of Theorem 9 and $\rho_{t} \in L^{2}\left(0, T ; H^{2}(\Omega)\right)$, the following error estimates hold for $h$ and $k$ small enough:

$$
\max _{0 \leq n \leq N-1}\left|\nabla e_{d, \rho}^{n+1}\right|^{2}+\sum_{n=0}^{N-1}\left|\nabla\left(e_{d, \rho}^{n+1}-e_{d, \rho}^{n}\right)\right|^{2}+\lambda k \sum_{n=0}^{N-1}\left|e_{d, \Delta}^{n+1}\right|^{2} \leq C\left(k^{2}+h^{2}\right)
$$

Proof Setting $\bar{\rho}_{h}=2 e_{d, \Delta}^{n+1}$ as a test function in the variational formulation (55), one arrives at

$$
2 k\left(\delta_{t} e_{d, \rho}^{n+1}, e_{d, \Delta}^{n+1}\right)+2 \lambda k\left|e_{d, \Delta}^{n+1}\right|^{2}+2\left(\zeta_{\rho}^{n+1}, e_{d, \Delta}^{n+1}\right)+2\left(\xi_{\rho}^{n+1}, e_{d, \Delta}^{n+1}\right)=0 .
$$

Integration by parts is justified in the first term on the left-hand side of (60) by taking $\bar{\rho}_{h}=e_{d, \rho}^{n+1}-e_{d, \rho}^{n}$ into (58) to obtain

$$
\begin{aligned}
2 k\left(e_{d, \Delta}^{n+1}, \delta_{t} e_{d, \rho}^{n+1}\right)= & 2 k\left(\nabla e_{d, \rho}^{n+1}, \nabla \delta_{t} e_{d, \rho}^{n+1}\right)=\left|\nabla e_{d, \rho}^{n+1}\right|^{2}-\left|\nabla e_{d, \rho}^{n}\right|^{2} \\
& +\left|\nabla\left(e_{d, \rho}^{n+1}-e_{d, \rho}^{n}\right)\right|^{2}
\end{aligned}
$$

Thus, incorporating (61) in (60), one has

$$
\begin{aligned}
& \left|\nabla e_{d, \rho}^{n+1}\right|^{2}-\left|\nabla e_{d, \rho}^{n}\right|^{2}+\left|\nabla\left(e_{d, \rho}^{n+1}-e_{d, \rho}^{n}\right)\right|^{2}+2 \lambda k\left|e_{d, \Delta}^{n+1}\right|^{2} \\
& \quad=-2\left(\zeta_{\rho}^{n+1}, e_{d, \Delta}^{n+1}\right)-2\left(\xi_{\rho}^{n+1}, e_{d, \Delta}^{n+1}\right)
\end{aligned}
$$

Next, we estimate the right-hand side of (62). Clearly, $\left(\xi_{\rho}^{n+1}, e_{d, \Delta}^{n+1}\right)$ has already bounded in the proof of Lemma 8 by replacing $e_{d, \rho}^{n+1}$ by $e_{d, \Delta}^{n+1}$. The only thing to be worth remaking on is the control of $\left(\xi_{\rho, 6}^{n+1}, e_{d, \Delta}^{n+1}\right)$. In fact, it is not hard to check

$$
\left(\xi_{\rho, 6}^{n+1}, e_{d, \Delta}^{n+1}\right) \leq C k^{3 / 2}\left\|\rho_{t}\right\|_{L^{2}\left(I_{n+1} ; H^{2}(\Omega)\right)}\left|\nabla e_{d, \rho}^{n+1}\right|
$$


Now, we focus on the control of $\left(\zeta_{\rho}^{n+1}, e_{d, \Delta}^{n+1}\right)$. In a totally analogous way to (41) we treat $\left(\zeta_{\rho, 1}^{n+1}, e_{d, \Delta}^{n+1}\right)$. We keep on with $\left(\zeta_{\rho, 1}^{n+1}, e_{d, \Delta}^{n+1}\right)$ as in (39). Hence, we have

$$
\left(\zeta_{\rho, 1}^{n+1}, e_{d, \Delta}^{n+1}\right)=k\left(\boldsymbol{e}_{d, \boldsymbol{u}}^{n} \cdot \nabla e_{d, \rho}^{n+1}, e_{d, \Delta}^{n+1}\right)+k\left(I_{h} \boldsymbol{u}\left(t_{n}\right) \cdot \nabla e_{d, \rho}^{n+1}, e_{d, \Delta}^{n+1}\right)
$$

The bound of the second term on the right-hand side of the foregoing decomposition takes advantage of the $\boldsymbol{L}^{\infty}$-regularity for the exact velocity as was done in (40). The most problematic term is $\left(\boldsymbol{e}_{d, \boldsymbol{u}}^{n} \cdot \nabla e_{d, \rho}^{n+1}, e_{d, \Delta}^{n+1}\right)$ which may be written as

$$
\begin{aligned}
\left(\boldsymbol{e}_{d, \boldsymbol{u}}^{n} \cdot \nabla e_{d, \rho}^{n+1}, e_{d, \Delta}^{n+1}\right)= & k\left(\boldsymbol{e}_{d, \boldsymbol{u}}^{n} \cdot \nabla\left(e_{d, \rho}^{n+1}-e^{n+1}(h)\right), e_{d, \Delta}^{n+1}\right) \\
& +k\left(\boldsymbol{e}_{d, \boldsymbol{u}}^{n} \cdot \nabla e^{n+1}(h), \Delta e^{n+1}(h)\right) \\
= & k\left(\boldsymbol{e}_{d, \boldsymbol{u}}^{n} \cdot \nabla\left(e_{d, \rho}^{n+1}-e^{n+1}(h)\right), e_{d, \Delta}^{n+1}\right) \\
& -k\left(\nabla \boldsymbol{e}_{d, \boldsymbol{u}}^{n}, \nabla e^{n+1}(h) \otimes \nabla e^{n+1}(h)\right) \\
& +\frac{k}{2}\left(\nabla \cdot \boldsymbol{e}_{d, \boldsymbol{u}}^{n},\left|\nabla e^{n+1}(h)\right|^{2}\right) \\
:= & \sum_{i=1}^{3} K_{i},
\end{aligned}
$$

where $\mathbf{a} \otimes \mathbf{b}$ denotes the tensorial product of two vectors $\boldsymbol{a}=\left(a_{i}\right)_{i=1}^{2}, \boldsymbol{b}=\left(b_{i}\right)_{i=1}^{2}$, a matrix with coefficients $(\boldsymbol{a} \otimes \boldsymbol{b})_{i, j}=a_{i} b_{j}$. Before estimating the terms $K_{i}$, note that from (50) one has, in particular, $\left|\nabla \boldsymbol{e}_{d, \boldsymbol{u}}^{n}\right|^{2} \leq C\left(k+h^{2} / k\right)$; hence $\left|\nabla \boldsymbol{e}_{d, \boldsymbol{u}}^{n}\right|^{2} \leq$ $C$ by using constraint $(S)$. Thus, the inverse inequality (17), Sobolev's inequalities $\|\nabla \rho\|_{L^{4}(\Omega)}^{2} \leq C|\nabla \rho|^{1 / 2}\|\nabla \rho\|_{L^{6}(\Omega)}^{3 / 2}$ and $\|\nabla \rho\|_{L^{6}(\Omega)} \leq C|\Delta \rho|$ for any $\rho \in H_{N, 0}^{2}(\Omega)$, and estimate (57) provide

$$
\begin{aligned}
K_{1} & \leq C k\left|\nabla \boldsymbol{e}_{d, \boldsymbol{u}}^{n}\right|\left|\nabla\left(\boldsymbol{e}_{d, \boldsymbol{u}}^{n+1}-e^{n+1}(h)\right)\right|\left\|e_{d, \Delta}^{n+1}\right\|_{L^{3}(\Omega)} \leq C k h^{1 / 2}\left|e_{d, \Delta}^{n+1}\right|^{2}, \\
K_{2}+K_{3} & \leq C k\left|\nabla \boldsymbol{e}_{d, \boldsymbol{u}}^{n}\right|\left\|\nabla e^{n+1}(h)\right\|_{L^{4}}^{2} \leq C k\left|\nabla \boldsymbol{e}_{d, \boldsymbol{u}}^{n}\right|\left|\nabla e^{n+1}(h)\right|^{1 / 2}\left|\Delta e^{n+1}(h)\right|^{3 / 2},
\end{aligned}
$$

hence by using the equality $-\Delta e^{n+1}(h)=e_{d, \Delta}^{n+1}$ owing to (56),

$$
K_{2}+K_{3} \leq C k\left|\nabla e^{n+1}(h)\right|^{1 / 2}\left|e_{d, \Delta}^{n+1}\right|^{3 / 2} .
$$

Next, we will see how to treat the term $\left|\nabla e^{n+1}(h)\right|$ entering into (63). This is factorized as follows:

$$
\begin{aligned}
\left|\nabla e^{n+1}(h)\right| & \leq\left|\nabla\left(e^{n+1}(h)-e_{d, \rho}^{n+1}\right)\right|+\left|\nabla e_{d, \rho}^{n+1}\right| \\
& \leq C h\left|e_{d, \Delta}^{n+1}\right|+\left|\nabla e_{d, \rho}^{n+1}\right|,
\end{aligned}
$$


where in the last estimate we have used estimate (57). Thus, by estimate (64) into (63), one gets

$$
K_{2}+K_{3} \leq C k\left|\nabla e_{d, \rho}^{n+1}\right|^{1 / 2}\left|e_{d, \Delta}^{n+1}\right|^{3 / 2}+C h^{1 / 2} k\left|e_{d, \Delta}^{n+1}\right|^{2} .
$$

Finally, Young's inequality applies to the previous bounds in (62) gives, for $h$ small enough,

$$
\begin{aligned}
& \left|\nabla e_{d, \rho}^{n+1}\right|^{2}-\left|\nabla e_{d, \rho}^{n}\right|^{2}+\left|\nabla\left(e_{d, \rho}^{n+1}-e_{d, \rho}^{n}\right)\right|^{2}+\lambda k\left|e_{d, \Delta}^{n+1}\right|^{2} \leq C k\left|\nabla e_{d, \rho}^{n+1}\right|^{2}+C k\left|\nabla \boldsymbol{e}_{d, \boldsymbol{u}}^{n}\right|^{2} \\
& \quad+C\left(h^{2}+k^{2}\right)\left\|\rho_{t}\right\|_{L^{2}\left(I_{n+1} ; H^{1}(\Omega)\right)}^{2}+C k^{2}\left(\left\|\boldsymbol{u}_{t}\right\|_{L^{2}\left(I_{n+1} ; H^{1}(\Omega)\right)}^{2}+\left\|\rho_{t}\right\|_{L^{2}\left(I_{n+1} ; H^{2}(\Omega)\right)}^{2}\right) \\
& \quad+C h^{2}\left(\|\boldsymbol{u}\|_{L^{2}\left(I_{n+1} ; H^{2}(\Omega)\right)}^{2}+\|\rho\|_{L^{2}\left(I_{n+1} ; H^{2}(\Omega)\right)}^{2}\right) .
\end{aligned}
$$

Hence, summing up (65) over $n$, using the regularity of the continuous solution in $(H 0)$, and the estimate $k \sum_{n=1}^{N}\left|\nabla \boldsymbol{e}_{d, \boldsymbol{u}}^{n}\right|^{2} \leq C\left(k^{2}+h^{2}\right)$ from Theorem 9, we arrive at (59) by applying the generalized discrete Gronwall's Lemma assuming $k$ small enough.

Finally, Theorem 3 can be proved from Theorem 11 simply by using the approximation properties (53) and

$$
\left|\rho-P_{h} \rho\right| \leq C h\|\rho\|_{H^{1}(\Omega)} \quad \forall \rho \in H^{1}(\Omega)
$$

(in particular, the extra approximation $\left|\nabla\left(\rho_{0}-\rho_{h}^{0}\right)\right| \leq C h\left\|\rho_{0}\right\|_{H^{2}(\Omega)}$ for the initial density $\rho_{0}$ holds).

\section{Two iterative methods with constant matrices}

The task in this section is to develop two iterative schemes, one for to approximate the density and the other one for the pair velocity-pressure, in such a way that the linear algebraic problems do not change of matrix at each iteration step, that is, the matrices are constant by iterations. The following iterative methods arises from approximating the nonlinear terms explicitly at a fixed step $n+1$ of problems (9) and (10)-(11).

Iterative method for problem (9). Known $\left(\rho_{h}^{n}, \boldsymbol{u}_{h}^{n}\right)$, the solution $\rho_{h}^{n+1}$ of (9) is approximated by the sequence $\left(\rho_{h}^{n+1, i}\right)_{i}$ defined as:

Initialization: Let $\rho_{h}^{n+1,0}=\rho_{h}^{n}$.

Step $i+1$ : Known $\rho_{h}^{n+1, i}$, find $\rho_{h}^{n+1, i+1} \in W_{h}$ such that for each $\bar{\rho}_{h} \in W_{h}$ :

$$
\left(\frac{\rho_{h}^{n+1, i+1}-\rho_{h}^{n}}{k}, \bar{\rho}_{h}\right)+\lambda\left(\nabla \rho_{h}^{n+1, i+1}, \nabla \bar{\rho}_{h}\right)=-\left(\boldsymbol{u}_{h}^{n} \cdot \nabla \rho_{h}^{n+1, i}, \bar{\rho}_{h}\right) .
$$

Iterative method for problem (10)-(11). Known $\left(\rho_{h}^{n}, \rho_{h}^{n+1}, \boldsymbol{u}_{h}^{n}\right)$, the solution $\left(\boldsymbol{u}_{h}^{n+1}\right.$, $\left.p_{h}^{n+1}\right)$ of (10)-(11) are approximated by the sequence $\left(\boldsymbol{u}_{h}^{n+1, i}, p_{h}^{n+1, i}\right)_{i}$ defined as: 
Initialization: Let $\boldsymbol{u}_{h}^{n+1,0}=\boldsymbol{u}_{h}^{n}$.

Step $i+1$ : Known $\boldsymbol{u}_{h}^{n+1, i}$, find $\left(\boldsymbol{u}_{h}^{n+1, i+1}, p_{h}^{n+1, i+1}\right) \in \boldsymbol{V}_{h} \times M_{h}$ such that for each $\left(\overline{\boldsymbol{u}}_{h}, \bar{p}_{h}\right) \in \boldsymbol{V}_{h} \times M_{h}$ :

$$
\left\{\begin{array}{l}
\left(\rho_{\widetilde{m}}^{\widetilde{M}} \frac{\boldsymbol{u}_{h}^{n+1, i+1}-\boldsymbol{u}_{h}^{n}}{k}, \overline{\boldsymbol{u}}_{h}\right)+\mu\left(\nabla \boldsymbol{u}_{h}^{n+1, i+1}, \nabla \overline{\boldsymbol{u}}_{h}\right)-\left(p_{h}^{n+1, i+1}, \nabla \cdot \overline{\boldsymbol{u}}_{h}\right) \\
=-\left(\left(\left(\rho_{h}^{n+1} \boldsymbol{u}_{h}^{n}-\lambda \nabla \rho_{h}^{n+1}\right) \cdot \nabla\right) \boldsymbol{u}_{h}^{n+1, i}, \overline{\boldsymbol{u}}_{h}\right)-\lambda \int_{\Omega}\left(\rho_{\tilde{m}}^{\widetilde{M}}-\rho_{h}^{n+1}\right)\left(\nabla \boldsymbol{u}_{h}^{n+1, i}\right)^{t}: \nabla \overline{\boldsymbol{u}}_{h} \\
-\frac{1}{2}\left(\nabla \cdot \boldsymbol{u}_{h}^{n} \rho_{h}^{n+1} \boldsymbol{u}_{h}^{n+1, i}, \overline{\boldsymbol{u}}_{h}\right)+\left(\rho_{h}^{n+1} \boldsymbol{f}^{n+1}, \overline{\boldsymbol{u}}_{h}\right)+\left(\left(\rho_{\widetilde{m}}^{\widetilde{M}}-\rho_{h}^{n}\right) \frac{\boldsymbol{u}_{h}^{n+1, i}-\boldsymbol{u}_{h}^{n}}{k}, \overline{\boldsymbol{u}}_{h}\right), \\
\left(\nabla \cdot \boldsymbol{u}_{h}^{n+1, i}, \bar{p}_{h}\right)=0 .
\end{array}\right.
$$

Next, we would like to prove that the approximations $\left(\rho_{h}^{n+1, i}, \boldsymbol{u}_{h}^{n+1, i}, p_{h}^{n+1, i}\right)$ converge to $\left(\rho_{h}^{n+1}, \boldsymbol{u}_{h}^{n+1}, p_{h}^{n+1}\right)$ as $i \rightarrow \infty$. For this, we define the consecutive differences:

$$
\begin{gathered}
\Phi_{i+1}=\boldsymbol{u}_{h}^{n+1, i+1}-\boldsymbol{u}_{h}^{n+1, i}, \Lambda_{i+1}=p_{h}^{n+1, i+1}-p_{h}^{n+1, i} \\
\text { and } \Psi_{i+1}=\rho_{h}^{n+1, i+1}-\rho_{h}^{n+1, i}
\end{gathered}
$$

which satisfy:

$$
\begin{aligned}
& \left(\frac{\Psi_{i+1}}{k}, \bar{\rho}_{h}\right)+\lambda\left(\nabla \Psi_{i+1}, \nabla \bar{\rho}_{h}\right)=-\left(\boldsymbol{u}_{h}^{n} \cdot \nabla \Psi_{i}, \bar{\rho}_{h}\right) . \\
& \int\left(\rho_{\tilde{m}}^{\widetilde{M}} \frac{\Phi_{i+1}}{k}, \overline{\boldsymbol{u}}_{h}\right)+\mu\left(\nabla \Phi_{i+1}, \nabla \overline{\boldsymbol{u}}_{h}\right)=-\left(\left(\left(\rho_{h}^{n+1} \boldsymbol{u}_{h}^{n}-\lambda \nabla \rho_{h}^{n+1}\right) \cdot \nabla\right) \Phi_{i}, \overline{\boldsymbol{u}}_{h}\right) \\
& \left\{-\lambda \int_{\Omega}\left(\rho_{\tilde{m}}^{\tilde{M}}-\rho_{h}^{n+1}\right)\left(\nabla \Phi_{i}\right)^{t}: \nabla \overline{\boldsymbol{u}}_{h}-\frac{1}{2}\left(\nabla \cdot \boldsymbol{u}_{h}^{n} \rho_{h}^{n+1} \Phi_{i}, \overline{\boldsymbol{u}}_{h}\right)\right. \\
& +\left(\Lambda_{i+1}, \nabla \cdot \overline{\boldsymbol{u}}_{h}\right)+\left(\left(\rho_{\widetilde{m}}^{\widetilde{M}}-\rho_{h}^{n+1}\right) \frac{\Phi_{i}}{k}, \overline{\boldsymbol{u}}_{h}\right), \\
& \left(\nabla \cdot \Phi_{i+1}, \bar{p}_{h}\right)=0 .
\end{aligned}
$$

Now, we take $\bar{\rho}_{h}=k \Psi_{i+1}$ as a test function in (66) and integrate by parts, obtaining

$$
\begin{aligned}
\left|\Psi_{i+1}\right|^{2}+\lambda k\left|\nabla \Psi_{i+1}\right|^{2} & =k\left(\Psi_{i} \boldsymbol{u}_{h}^{n}, \nabla \Psi_{i+1}\right)+k\left(\nabla \cdot \boldsymbol{u}_{h}^{n} \Psi_{i}, \Psi_{i+1}\right) \\
& \leq C k\left\|\boldsymbol{u}_{h}^{n}\right\|_{H^{1}(\Omega)}^{2}\left\|\Psi_{i}\right\|_{L^{3}(\Omega)}^{2}+\frac{1}{2} \lambda k\left|\nabla \Psi_{i+1}\right|^{2} \\
& \leq C k\left|\Psi_{i}\right|\left|\nabla \Psi_{i}\right|+\frac{1}{2} k\left|\nabla \Psi_{i+1}\right|^{2} .
\end{aligned}
$$

Then

$$
\left|\Psi_{i+1}\right|^{2}+\frac{\lambda}{2} k\left|\nabla \Psi_{i+1}\right|^{2} \leq C k^{1 / 2}\left(\left|\Psi_{i}\right|^{2}+\frac{\lambda}{2} k\left|\nabla \Psi_{i}\right|^{2}\right) .
$$


Setting $k$ small enough such that $\alpha:=C k^{1 / 2}<1$ and applying the Banach fixed point theorem, we have that $\left\{\rho^{n+1, i}\right\}_{i}$ is a Cauchy sequence in $H^{1}(\Omega)$; hence $\rho_{h}^{n+1, i} \rightarrow \rho_{h}^{n+1}$ in $H^{1}(\Omega)$-strong, as $i \rightarrow+\infty$, with rate of convergence $\alpha^{i}$ (in [6] this technique is used in order to decouple an scheme for a nematic liquid crystal model).

On the other hand, we take $\overline{\boldsymbol{u}}_{h}=k \Phi_{i+1}$ in (67) and $\bar{p}_{h}=k \Lambda_{i+1}$ in (68), getting

$$
\left\{\begin{array}{l}
\rho_{\tilde{m}}^{\widetilde{M}}\left|\Phi_{i+1}\right|^{2}+\mu k\left|\nabla \Phi_{i+1}\right|^{2}=-k\left(\left(\left(\rho_{h}^{n+1} \boldsymbol{u}_{h}^{n}-\lambda \nabla \rho_{h}^{n+1}\right) \cdot \nabla\right) \Phi_{i}, \Phi_{i+1}\right) \\
-\lambda k \int_{\Omega}\left(\rho_{\widetilde{m}}^{\widetilde{M}}-\rho_{h}^{n+1}\right)\left(\nabla \Phi_{i}\right)^{t}: \nabla \Phi_{i+1}+\left(\left(\rho_{\widetilde{m}}^{\widetilde{M}}-\rho_{h}^{n+1}\right) \Phi_{i}, \Phi_{i+1}\right) \\
-\frac{k}{2}\left(\nabla \cdot \boldsymbol{u}_{h}^{n} \rho_{h}^{n+1} \Phi_{i}, \Phi_{i+1}\right):=F_{1}+F_{2}+F_{3}+F_{4} .
\end{array}\right.
$$

Using that $\left|\rho_{\widetilde{m}}^{\widetilde{M}}-\rho_{h}^{n+1}\right| \leq(\widetilde{M}-\widetilde{m}) / 2$ in $\Omega$ and admitting the following additional hypothesis for the scheme

$$
\left\|\rho_{h}^{n+1} \boldsymbol{u}_{h}^{n}-\lambda \nabla \rho_{h}^{n+1}\right\|_{L^{6}(\Omega)} \leq C
$$

we can bound

$$
\begin{aligned}
F_{1} \leq & C k\left|\nabla \Phi_{i}\right|\left\|\Phi_{i+1}\right\|_{L^{3}(\Omega)} \leq \frac{\delta}{2}\left(\frac{\rho_{\tilde{m}}^{\tilde{M}}}{2}\left|\Phi_{i+1}\right|^{2}+\mu_{1} k\left|\nabla \Phi_{i+1}\right|^{2}\right) \\
& +C_{\delta} k^{1 / 2} \mu_{1} k\left|\nabla \Phi_{i}\right|^{2},
\end{aligned}
$$

where $\mu_{1}=\mu-\frac{\lambda}{2} \frac{\widetilde{M}-\widetilde{m}}{2}>0[$ see (13)],

$$
\begin{aligned}
& F_{2} \leq \lambda \frac{\widetilde{M}-\widetilde{m}}{2} k\left|\nabla \Phi_{i}\right|\left|\nabla \Phi_{i+1}\right| \leq \lambda \frac{\widetilde{M}-\widetilde{m}}{2} k\left(\frac{1}{2}\left|\nabla \Phi_{i}\right|^{2}+\frac{1}{2}\left|\nabla \Phi_{i+1}\right|^{2}\right), \\
& F_{3} \leq \frac{\widetilde{M}-\widetilde{m}}{2}\left|\Phi_{i}\right|\left|\Phi_{i+1}\right| \leq\left(\frac{\widetilde{M}-\widetilde{m}}{2}\right)^{2} \frac{1}{2 \rho_{\tilde{m}}^{\widetilde{M}}}\left|\Phi_{i}\right|^{2}+\frac{\rho_{\tilde{m}}^{\widetilde{M}}}{2}\left|\Phi_{i+1}\right|^{2} .
\end{aligned}
$$

Again, as $\left|\left(\nabla \cdot \boldsymbol{u}_{h}^{n}\right) \rho_{h}^{n+1}\right| \leq C$ we can bound $F_{4}$ as $F_{1}$ :

$$
\begin{aligned}
F_{4} \leq C k\left\|\Phi_{i}\right\|_{L^{6}(\Omega)}\left\|\Phi_{i+1}\right\|_{L^{3}(\Omega)} \leq & \frac{\delta}{2}\left(\frac{\rho_{\tilde{m}}^{\widetilde{M}}}{2}\left|\Phi_{i+1}\right|^{2}+\mu_{1} k\left|\nabla \Phi_{i+1}\right|^{2}\right) \\
& +C_{\delta} k^{1 / 2} \mu_{1} k\left|\nabla \Phi_{i}\right|^{2}
\end{aligned}
$$


Applying these estimates in (69), we get

$$
\begin{aligned}
(1-\delta)\left(\frac{\rho_{\widetilde{M}}^{\widetilde{M}}}{2}\left|\Phi_{i+1}\right|^{2}+\mu_{1} k\left|\nabla \Phi_{i+1}\right|^{2}\right) \leq & \left(\frac{\widetilde{M}-\widetilde{m}}{\widetilde{M}+\widetilde{m}}\right)^{2} \frac{\rho_{\widetilde{m}}^{\widetilde{M}}}{2}\left|\Phi_{i}\right|^{2} \\
& +\left(C_{\delta} k^{1 / 2}+\frac{\lambda}{2 \mu_{1}} \frac{\widetilde{M}-\widetilde{m}}{2}\right) \mu_{1} k\left|\nabla \Phi_{i}\right|^{2} .
\end{aligned}
$$

Observe that $\frac{\lambda}{2 \mu_{1}} \frac{\widetilde{M}-\tilde{m}}{2}<1$, i.e. $\frac{\lambda}{2} \frac{\tilde{M}-\tilde{m}}{2}<\mu_{1}$, by the definition of $\mu_{1}$ given in (13). Hence, choosing $C_{\delta} k^{1 / 2}$ small enough such that $C_{\delta} k^{1 / 2}+\frac{\lambda}{2 \mu_{1}} \frac{\tilde{M}-\tilde{m}}{2}<1$ and $\delta$ small enough such that $(1-\delta)>\max \left\{\left(\frac{\tilde{M}-\tilde{m}}{\widetilde{M}+\widetilde{m}}\right)^{2}, C_{\delta} k^{1 / 2}+\frac{\lambda}{2 \mu_{1}} \frac{\tilde{M}-\tilde{m}}{2}\right\}$, we arrive at the recursive inequality

$$
\left(\frac{\rho_{\widetilde{m}}^{\widetilde{M}}}{2}\left|\Phi_{i+1}\right|^{2}+\mu_{1} k\left|\nabla \Phi_{i+1}\right|^{2}\right) \leq \widetilde{\alpha}\left(\frac{\rho_{\widetilde{m}}^{\widetilde{M}}}{2}\left|\Phi_{i}\right|^{2}+\mu_{1} k\left|\nabla \Phi_{i}\right|^{2}\right),
$$

where $\widetilde{\alpha}=\frac{1}{1-\delta} \max \left\{\left(\frac{\widetilde{M}-\widetilde{m}}{\widetilde{M}+\widetilde{m}}\right)^{2}, C_{\delta} k^{1 / 2}+\frac{\lambda}{2 \mu_{1}} \frac{\widetilde{M}-\widetilde{m}}{2}\right\}$. Since $\widetilde{\alpha}<1$, we extract the same convergence result that for the density, that is, $\boldsymbol{u}^{n+1, i} \rightarrow \boldsymbol{u}_{h}^{n+1}$ in $H^{1}(\Omega)$ as $i \rightarrow+\infty$. Finally, by using the inf-sup condition, we can deduce that $p_{h}^{n+1, i} \rightarrow p_{h}^{n+1}$ in $L^{2}(\Omega)$ as $i \rightarrow+\infty$.

Consequently, we have arrived at the following result

Theorem 12 Admitting $k$ small enough and the stability estimates $0 \leq \widetilde{m} \leq \rho_{h}^{n+1} \leq$ $\tilde{M},\left\|\boldsymbol{u}_{h}^{n}\right\|_{L^{6}(\Omega)} \leq C$ and $\left\|\nabla \rho_{h}^{n+1}\right\|_{L^{6}(\Omega)} \leq C$ (see (70)), where $C>0$ is a constant independent of $h$ and $k$, then the iterative methods (14) and (15)-(16) converge towards the unique solution of scheme (9) and (10)-(11), respectively. More concretely, one finds the convergences $\rho_{h}^{n+1, i} \rightarrow \rho_{h}^{n+1}$ in $H^{1}(\Omega), \boldsymbol{u}^{n+1, i} \rightarrow \boldsymbol{u}_{h}^{n+1}$ in $H^{1}(\Omega)$ and $p_{h}^{n+1, i} \rightarrow p_{h}^{n+1}$ in $L^{2}(\Omega)$ as $i \rightarrow+\infty$.

Finally, note that the uniform bounds (70) imposed on the scheme in Theorem 12 can be deduced from the error estimates obtained in the previous section. Indeed, from the error estimates $k \sum_{n=0}^{N-1}\left|\nabla \boldsymbol{e}_{\boldsymbol{u}}^{n+1}\right|^{2} \leq C\left(h^{2}+k\right)$ and $k \sum_{n=0}^{N-1}\left|\boldsymbol{e}_{\Delta}^{n+1}\right|^{2} \leq C\left(h^{2}+k\right)$ we have in particular the uniform estimates $\left|\nabla \boldsymbol{u}_{h}^{n+1}\right| \leq C$ and $\left|\Delta_{h} \rho_{h}^{n+1}\right| \leq C$ (the constraint (S) implies in particular $h^{2} / k \leq C$ ). By considering the Sobolev embedding $\left\|\boldsymbol{u}_{h}^{n}\right\|_{L^{6}(\Omega)} \leq C\left|\nabla \boldsymbol{u}_{h}^{n}\right| \leq C$, it suffices to prove that $\left\|\nabla \rho_{h}^{n+1}\right\|_{L^{6}(\Omega)} \leq C\left|\Delta_{h} \rho_{h}^{n+1}\right|$. Indeed,

$$
\begin{aligned}
\left\|\nabla \rho_{h}^{n+1}\right\|_{L^{6}(\Omega)} \leq & \left\|\nabla\left(\rho_{h}^{n+1}-K_{h} \rho^{n+1}(h)\right)\right\|_{L^{6}(\Omega)}+\left\|\nabla\left(K_{h} \rho^{n+1}(h)-\rho^{n+1}(h)\right)\right\|_{L^{6}(\Omega)} \\
& +\left\|\nabla \rho^{n+1}(h)\right\|_{L^{6}(\Omega)},
\end{aligned}
$$


where $\rho^{n+1}(h) \in H_{N}^{2}(\Omega)$ solves the problem

$$
-\Delta \rho^{n+1}(h)=-\Delta_{h} \rho_{h}^{n+1} \text { in } \Omega,\left.\quad \frac{\partial \rho^{n+1}(h)}{\partial \boldsymbol{n}}\right|_{\partial \Omega}=0, \quad \int_{\Omega} \rho^{n+1}(h)=0,
$$

which offers us the property $\left\|\rho^{n+1}(h)\right\|_{H^{2}(\Omega)} \leq C\left|\Delta_{h} \rho_{h}^{n+1}\right|$ by hypothesis $(H 1)$. Using the inverse inequality $\left\|\nabla \bar{\rho}_{h}\right\|_{L^{6}(\Omega)} \leq C h^{-1}\left\|\bar{\rho}_{h}\right\|_{H^{1}(\Omega)}$ and the approximation property $\left|\nabla\left(\rho_{h}^{n+1}-\rho^{n+1}(h)\right)\right| \leq C h\left|\Delta_{h} \rho_{h}^{n+1}\right|$ analogous to (57), we bound

$$
\begin{aligned}
& \left\|\nabla\left(\rho_{h}^{n+1}-K_{h} \rho^{n+1}(h)\right)\right\|_{L^{6}(\Omega)} \leq C h^{-1}\left\|\rho_{h}^{n+1}-K_{h}\left(\rho^{n+1}(h)\right)\right\|_{H^{1}(\Omega)} \leq C\left|\Delta_{h} \rho_{h}^{n+1}\right|, \\
& \left\|\nabla\left(\rho^{n+1}(h)-K_{h} \rho^{n+1}(h)\right)\right\|_{L^{6}(\Omega)} \leq C\left\|\rho^{n+1}(h)\right\|_{H^{2}(\Omega)} \leq C\left|\Delta_{h} \rho_{h}^{n+1}\right|, \\
& \left\|\nabla \rho^{n+1}(h)\right\|_{L^{6}(\Omega)} \leq C\left\|\rho^{n+1}(h)\right\|_{H^{2}(\Omega)} \leq C\left|\Delta_{h} \rho_{h}^{n+1}\right| ;
\end{aligned}
$$

hence $\left\|\nabla \rho_{h}^{n+1}\right\|_{L^{6}(\Omega)} \leq C\left|\Delta_{h} \rho_{h}^{n+1}\right|$.

\section{References}

1. Antontsev, S.N., Kazhikhov, A.V., Monakhov, V.N.: Boundary value problems in mechanics of nonhomogeneous fluids. In: Studies in Mathematical and its Applications, vol. 22. North-Holland, Amsterdam (1990)

2. Berião da Veiga, H.: Diffusion on viscous fluids, existence and asymptotic properties of solutions. Ann. Sc. Norm. Sup. Pisa 10, 341-355 (1983)

3. Brenner, S., Scott, L.R.: The mathematical theory of finite element methods. In: Texts in Appled Mathematics, vol. 15. Springer-Verlag, Berlin (1994)

4. Ciarlet, P.G.: The Finite Element Method for Elliptic Problems. North-Holland, Amsterdam (1987)

5. Ètienne, J., Saramito, P.: A priori error estimates of the Lagrange-Galerkin method for KazhikhovSmagulov type systems. C. R. Math. Acad. Sci. Paris 341(12), 769-774 (2005)

6. Girault, V., Guillén-González, F.: Mixed formulation, approximation and decoupling algorithm for a nematic liquid crystals model. Math. Comput. (to appear)

7. Girault, V., Raviart, P.A.: Finite Element Methods for Navier-Stokes Equations: Theory and Algorithms. Springer-Verlag, Berlin (1986)

8. Guillén-González, F., Damázio, P., Rojas-Medar, M.A.: Approximation by an iterative method for regular solutions for incompressible fluids with mass diffusion. J. Math. Anal. Appl. 326(1), 468-487 (2007)

9. Guillén-González, F., Gutiérrez-Santacreu, J.V.: Unconditional stability and convergence of fully discrete schemes for $2 D$ viscous fluids models with mass diffusion. Math. Comput. 77(263), 1495-1524 (2008a)

10. Guillén-González, F., Gutiérrez-Santacreu, J.V.: Conditional stability and convergence of a fully discrete scheme for $3 D$ Navier-Stokes equations with mass diffusion. SIAM J. Numer. Anal. 46(5), 2276-2308 (2008b)

11. Guillén-González, F., Gutiérrez-Santacreu, J.V.: Stability and convergence for a complete model of mass diffusion. Appl. Num. Math. (to appear)

12. Heywood, J.G., Rannacher, R.: Finite element approximation of the nonstationary Navier-Stokes problem. Part I: regularity of solutions and second-order error estimates for spatial discretization. SIAM J. Numer. Anal. 19(2), 275-311 (1982)

13. Kazhikhov, A., Smagulov, S.: The correctness of boundary value problems in a diffusion model of an inhomogeneous fluid. Sov. Phys. Dokl. 22(1), 249-252 (1977)

14. Salvi, R.: On the existence of weak solutions of boundary-value problems in a diffusion model of an inhomogeneous liquid in regions with moving boundaries. Portugaliae Math. 43, 213-233 (1986) 
15. Salvi, R.: On the existence and regularity of the solutions to incompressible Navier-Stokes equations in presence of difussion parabolic and Navier-Stokes equations. Banach Center Publications, vol. 81, pp. 383-419 (2008)

16. Secchi, P.: On the motion of viscous fluids in the precense of diffusion. SIAM J. Math. Anal. 19, 22-31 (1988)

17. Secchi, P.: On the inicial value problem for the equations of motion of viscous incompressible fluids in the presence of diffusion. Bollettino U.M.I., 6 1-B, pp. 117-1130 (1982) 